\title{
Protist community composition during early phytoplankton blooms in the naturally iron-fertilized Kerguelen area (Southern Ocean)
}

\author{
C. Georges, S. Monchy, S. Genitsaris, and U. Christaki \\ INSU-CNRS, UMR8187 LOG, Laboratoire d'Océanologie et de Géosciences, Université du Littoral Côte d'Opale, \\ ULCO, 32 avenue Foch, 62930 Wimereux, France \\ Correspondence to: U. Christaki (urania.christaki@univ-littoral.fr)
}

Received: 18 June 2014 - Published in Biogeosciences Discuss.: 21 July 2014

Revised: 16 September 2014 - Accepted: 17 September 2014 - Published: 21 October 2014

\begin{abstract}
Microbial eukaryotic community composition was examined by $18 \mathrm{~S}$ rRNA gene tag pyrosequencing, during the early phase of spring phytoplankton blooms induced by natural iron fertilization, off Kerguelen Island in the Southern Ocean (KEOPS2 cruise). A total of 999 operational taxonomical units (OTUs), affiliated to 30 known high-level taxonomic groups, were retrieved from 16 samples collected in the upper $300 \mathrm{~m}$ water column. The alveolata group was the most abundant in terms of sequence number and diversity (696 OTUs). The majority of alveolata sequences were affiliated to Dinophyceae and to two major groups of marine alveolates (MALV-I and MALV-II). In the upper $180 \mathrm{~m}$, only $13 \%$ of the OTUs were shared between of the fertilized stations and the reference site characterized by highnutrient low-chlorophyll (HNLC) waters. Fungi and Cercozoa were present in iron-fertilized waters, but almost absent in the HNLC samples, while Haptophyta and Chlorophyta characterized the HNLC sample. Finally, the $300 \mathrm{~m}$ depth samples of all stations were differentiated by the presence of MALV-II and Radiolaria. Multivariate analysis, examining the level of similarity between different samples, showed that protistan assemblages differed significantly between the HNLC and iron-fertilized stations, but also between the diverse iron-fertilized blooms.
\end{abstract}

\section{Introduction}

Molecular investigations into the planktonic protists of natural microbial communities have revealed an astonishing diversity (e.g. Caron et al., 2012 and references therein) and a variety of novel and/or previously unobserved groups of saprophytes, parasites and intracellular symbionts (e.g Guillou et al., 2008; Massana and Pedrós-Alió, 2008; Bråte et al., 2012). The wide ecological roles of protists include: phototrophic and mixotrophic species, belonging to the primary producers; heterotrophic species, acting as a "link" between the microbial food web and the higher trophic levels, as well as decomposers and parasitic taxa (Caron et al., 2009 and references therein). A series of molecular studies have examined spatial or temporal patterns in protistan community structure and diversity. These have indicated that the microbial community structure is generally highly responsive to environmental forcing, and that dominant protistan taxa can differ markedly over temporal and spatial scales associated with common oceanographic features (e.g Countway et al., 2007, 2010; Nolte et al., 2010; Gilbert et al., 2012; Mangot et al., 2013; Lie et al., 2013; Wolf et al., 2014; Christaki et al., 2014).

The Southern Ocean has a unique geography with several large-scale water masses separated by oceanic fronts, and has major implications for the global ocean circulation and climate system. It is also the largest high-nutrient lowchlorophyll (HNLC) ocean, in which iron limits phytoplankton production, resulting in a large stock of major inorganic nutrients (Martin and Fitzwater, 1990). A pronounced shift to larger phytoplankton cells, in particular diatoms, has been generally observed resulting in natural (Blain et al., 2007; Pollard et al., 2009) or artificial (Boyd et al., 2007; Smetacek et al., 2012) iron additions. While evidence of iron limitation of phytoplankton growth is unequivocal, the subsequent direct or indirect impact of iron on heterotrophic eukaryotes of the microbial food web is less clear. For example, a moderate increase in microzooplankton biomass was 
Table 1. Brief description of the stations. The depth of the mixed layer (ML) is based on a difference in sigma of 0.02 to the surface value. The mean ML $( \pm \mathrm{SD})$ of all CTD casts performed during the occupation of the stations is given. Ze: the euphotic layer depth. For Chl $a$ and major inorganic nutrients mean values $\pm \mathrm{SD}$ for the mixed layer.

\begin{tabular}{|c|c|c|c|c|c|c|c|c|c|c|c|c|}
\hline Station & $\begin{array}{l}\text { Date } \\
(2011)\end{array}$ & $\begin{array}{l}\text { Latitude } \\
\left({ }^{\circ} \mathrm{N}\right)\end{array}$ & $\begin{array}{l}\text { Longitude } \\
\left({ }^{\circ} \mathrm{E}\right)\end{array}$ & $\begin{array}{l}\text { Station depth } \\
(\mathrm{m})\end{array}$ & $\begin{array}{l}\text { Sampling depths } \\
\text { (m) }\end{array}$ & $\begin{array}{l}\text { ML } \\
\text { (m) }\end{array}$ & $\begin{array}{l}\mathrm{Ze} \\
(\mathrm{m})\end{array}$ & $\begin{array}{l}\text { Chl } a \\
\left(\mu g \mathrm{~L}^{-1}\right)^{\mathrm{a}}\end{array}$ & $\begin{array}{l}\mathrm{NO}_{3}+\mathrm{NO}_{2} \\
(\mu \mathrm{M})^{\mathrm{b}}\end{array}$ & $\begin{array}{l}\mathrm{PO}_{4} \\
(\mu \mathrm{M})^{\mathrm{b}}\end{array}$ & $\begin{array}{l}\mathrm{Si}(\mathrm{OH})_{4} \\
(\mu \mathrm{M})^{\mathrm{c}}\end{array}$ & $\begin{array}{l}\mathrm{DFe} \\
(\mathrm{nM})^{\mathrm{d}}\end{array}$ \\
\hline $\mathrm{R}-2$ & 26 Oct & -50.359 & 66.717 & 2450 & $20,60,150,300$ & $105 \pm 15$ & 92 & $0.25 \pm 0.08$ & $26.0 \pm 0.2$ & $1.83 \pm 0.03$ & $12.3 \pm 0.3$ & $0.08 \pm 0.07$ \\
\hline F-L & $7 \mathrm{Nov}$ & -48.505 & 74.614 & 2690 & $20,65,180,300$ & $38 \pm 7$ & 28 & $4.00 \pm 1.58$ & $20.5 \pm 1.9$ & $1.06 \pm 0.21$ & $7.7 \pm 0.8$ & $0.22 \pm 0.06$ \\
\hline E-4W & $10 \mathrm{Nov}$ & -48.765 & 71.425 & 1398 & $30,80,150,300$ & $61 \pm 11$ & 31 & $2.38 \pm 0.31$ & $25.4 \pm 1.0$ & $1.79 \pm 0.10$ & $18.5 \pm 1.2$ & $0.17 \pm 0.03$ \\
\hline A3-2 & $16 \mathrm{Nov}$ & -50.624 & 72.056 & 528 & $20,80,160,300$ & $153 \pm 15$ & 38 & $2.03 \pm 0.33$ & $26.2 \pm 0.4$ & $1.78 \pm 0.03$ & $18.9 \pm 0.5$ & $0.16 \pm 0.03$ \\
\hline
\end{tabular}

${ }^{\mathrm{a}}$ Lasbleiz et al. (this volume), ${ }^{\mathrm{b}}$ Blain et al. (this volume), ${ }^{\mathrm{c}}$ Closset et al. (this volume), ${ }^{\mathrm{d}}$ Quéroué et al. (unpublished KEOPS2 data).

observed during the iron fertilization experiment IronEx-2 in the Equatorial Pacific sector and the SOIREE in the Southern Ocean (Landry et al., 2000; Hall and Safi, 2001). In contrast, the microzooplankton grazing pressure on the total phytoplankton community decreased during the iron fertilization experiment SERIES in the Gulf of Alaska and the SEEDS1 in the western subarctic Pacific (Boyd et al., 2004; Saito et al., 2005). In the Kerguelen region, the iron limitation of the Southern Ocean is relieved by natural iron fertilization (Blain et al., 2007). Natural iron fertilization is an uncommon process in which iron supply of the surface waters from iron-rich deep water is observed. Only two studies referred to natural iron fertilization in the vicinity of Crozet (Pollard et al., 2009) and Kerguelen Islands (Blain et al., 2007). The KEOPS 1 cruise demonstrated that the phytoplankton bloom was sustained by iron supply from iron-rich deep water below, representing natural iron fertilization (Blain et al., 2007). This study also showed that microzooplankton grazing was an important factor for phytoplankton biomass decrease in the bloom area (Brussaard et al., 2008) mainly affecting the small-sized phytoplankton population (Brussaard et al., 2008; Christaki et al., 2008).

The KEOPS2 cruise sampling strategy covered spatially diverse iron-fertilized stations at early bloom stages in the Kerguelen plateau and ocean region (OctoberNovember 2011). These data showed that natural iron fertilization of the Southern Ocean on the scale of hundreds of thousands of square kilometres produced a mosaic of blooms, and that the biological and biogeochemical response to fertilization was diverse.

The objective of this study was to explore the microbial eukaryotic community structure using $18 \mathrm{~S}$ rRNA gene tag pyrosequencing during the onset of spring phytoplankton blooms in the context of natural iron fertilization of the Southern Ocean. The hypothesis tested was that the protistan communities would differ between the blooms, and between the iron-fertilized blooms and the HNLC waters. The use of tag pyrosequencing provided a unifying approach for assessing the breadth of protistan communities, including the groups that are quasi-impossible to characterize using traditional approaches of microscopy and culture (e.g. MAST, MALV, Fungi, and others).

\section{Materials and methods}

\subsection{Sample collection and DNA extraction}

The present study was carried out during the KEOPS2 cruise from October 15 to 20 November 2011. Water samples were collected from four stations above and off the Kerguelen plateau (Fig. 1a, b). Stations A3-2, E-4W, and F-L were located in the blooms, while the reference station R-2 was located in the HNLC region (Fig. 1a, b). All water samples were collected with $12 \mathrm{~L}$ Niskin bottles mounted on a rosette equipped with a CTDO Seabird SBE911-plus. According to CTD profiles, four sampling depths were chosen at each station in order to represent the mixed layer (ML), the bottom of the ML, and the deeper waters (Table 1). Five to 7.5 litres of each depth were subsequently filtered on 10,3 , and $0.6 \mu \mathrm{m}$, $47 \mathrm{~mm}$ nucleopore filters (Whatman, USA) using a serial filtration system at very low pressure $(15 \mathrm{rpm})$. The serial filtration was performed in order to avoid filter clumping and to minimize disruption of fragile protists. The filters were immediately frozen in liquid nitrogen and then stored at $-80^{\circ} \mathrm{C}$ until analysis. After pooling together and cutting into small pieces the 10,3 , and $0.6 \mu \mathrm{m}$ filters, DNA extractions were carried out using the MO BIO PowerWater DNA Isolation Kit (MO BIO Laboratories, Inc, Carlsbad, CA), following the manufacturer's protocol instructions.

\subsection{PCR and tag pyrosequencing}

The DNA samples were amplified using the two universal eukaryote primers $18 \mathrm{~S}-82 \mathrm{~F}$ ( $5^{\prime}$-GAAACTGCGAATGGCTC$3^{\prime}$, López-Garcia et al., 2003) and Euk-516r (5'ACCAGACTTGCCCTCC-3', Amann et al., 1990). These primers have been designed to amplify the variable V2 and V3 eukaryote $18 \mathrm{~S}$ rRNA gene regions. A $10 \mathrm{bp}$ tag sequence specific to each sample, a 4 bp TCAG key, and a $26 \mathrm{bp}$ adapter for the GS FLX technology, were added to the primers. Polymerase chain reactions were carried out according to standard conditions for Platinum Tag High-Fidelity DNA polymerase (Invitrogen) with $10 \mathrm{ng}$ of environmental DNA as a template. After the denaturation step at $94^{\circ} \mathrm{C}$ for $2 \mathrm{~min}, 30$ cycles of amplification were performed with a GeneAmp PCR System Apparatus (Applied 
(a)

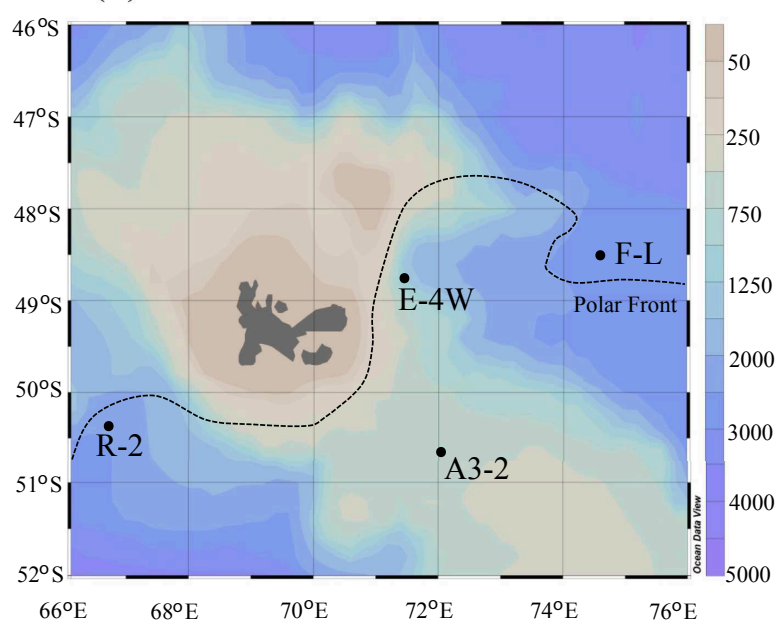

(b)

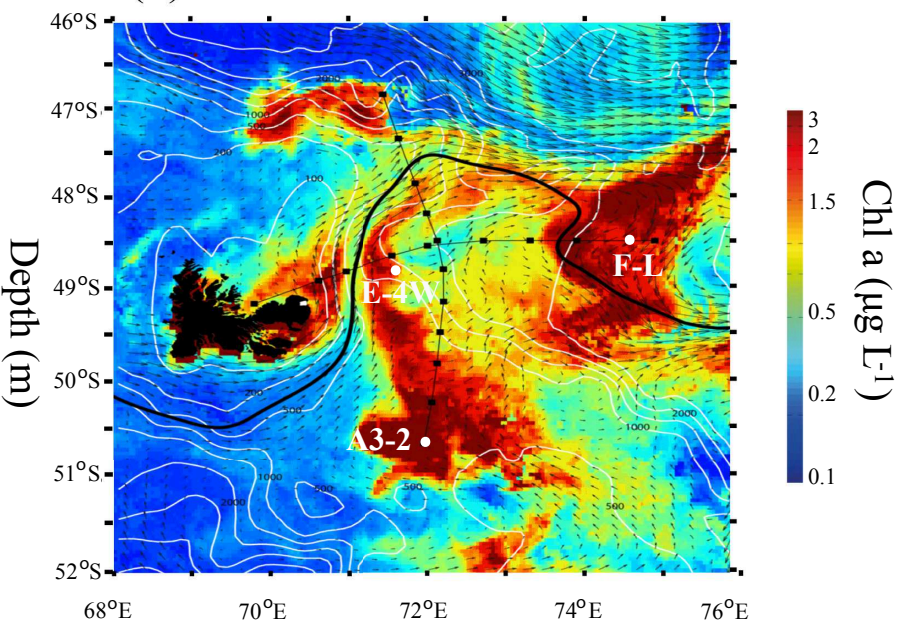

Figure 1. Bathymetry of the study area and location of the sampled stations (a), and Chl $a$ (colour scale), surface velocity fields (arrows), the polar front (PF, black line) (b). The chlorophyll content represented on the map corresponds to the last week of the KEOPS2 and the cross indicates the position of the north-south and east-west transects sampled to provide an overview of the blooms. Map is courtesy of Y. Park and colleagues.

Biosystems) as follows: $15 \mathrm{~s}$ at $94^{\circ} \mathrm{C}, 30 \mathrm{~s}$ at $50^{\circ} \mathrm{C}, 1 \mathrm{~min}$ at $72^{\circ} \mathrm{C}$, and $7 \mathrm{~min}$ at $72^{\circ} \mathrm{C}$. Tag pyrosequencing was carried out by the company GenoScreen (Lille, France). The library was prepared following the procedures described by Roche (Basel, Switzerland) and used in a 1/4 plate run on a 454 GS FLX Titanium sequencer. Pyrosequences were submitted on GenBank-SRA under the accession number SRP041236.

\subsection{Quality filtering and taxonomic affiliations of the sequences}

The sequences were processed using the MOTHUR 1.28.0 software (Schloss, 2009) following the standard operating procedure (http://www.mothur.org/wiki/Schloss_SOP) (Schloss et al., 2011). First, flowgrams were extracted and demultiplexed according to their tag. The resulting 16 flowgrams were denoised using the MOTHUR 1.28.0 implementation of PyroNoise (Quince, 2009). Primer sequences, TAG and key fragments were subsequently removed, and only sequences above $200 \mathrm{bp}$ long, displaying less than eight homopolymers, were kept. The remaining sequences were dereplicated to unique sequences and aligned against the SILVA 108 database (http://www.arb-silva.de/) containing 62587 eukaryotes SSU-18S rRNA sequences. Around $7 \%$ of the sequences suspected of being chimeras were removed using the UCHIME software (http://drive5.com/ usearch/manual/uchime_algo.html) (Edgar, 2011). The remaining sequences were clustered into operational taxonomical units (OTUs) at $97 \%$ similarity threshold. Single singletons (unique amplicons after $97 \%$ clustering that occurred exclusively in only one sample) were removed from downstream analyses, as these are most likely erroneous sequenc- ing products (Reeder and Knight, 2009; Kunin et al., 2010; Behnke et al., 2010). This data set showed a representative overview of the diversity as indicated by the rarefaction curves reaching a plateau in most cases (Supplement Fig. S1). All OTUs were given a putative taxonomic affiliations based on BLAST (Altschul et al., 1990) identification of the closest cultured or uncultured relatives against the PR2 (Guillou et al., 2013) and the GenBank databases. The OTUs identified as metazoan were removed from downstream analysis. However, the metazoan OTUs displayed high and heterogeneous number of sequences between samples, making subsampling of the remaining OTUs unsuitable as it resulted in a drastic loss of diversity. For this reason, the data are presented based on the relative abundance of OTUs in each sample.

\subsection{Data analysis}

Rarefaction curves and alpha diversity estimators within particular samples (richness estimator $S_{\text {Chaol }}$; the heterogeneity of the diversity; Simpson and Berger-Parker indices) were calculated with the PAST 2.17c software (Hammer et al., 2001). The $S_{\text {Chao1 }}$ approach uses the numbers of singletons and doubletons to estimate the number of expected species. According to $S_{\text {Chaol }}$, "missing" species information is mostly concentrated on those of low frequency counts. The Simpson index measures the "evenness" of the community and ranges from 0 (one taxon dominates the community) to 1 (all taxa are represented equally). Berger-Parker indicates the relative abundance of the dominant OTU in each sample (for more details, see Magurran, 2004). Protistan assemblages, from the different samples, were compared using the Plymouth 

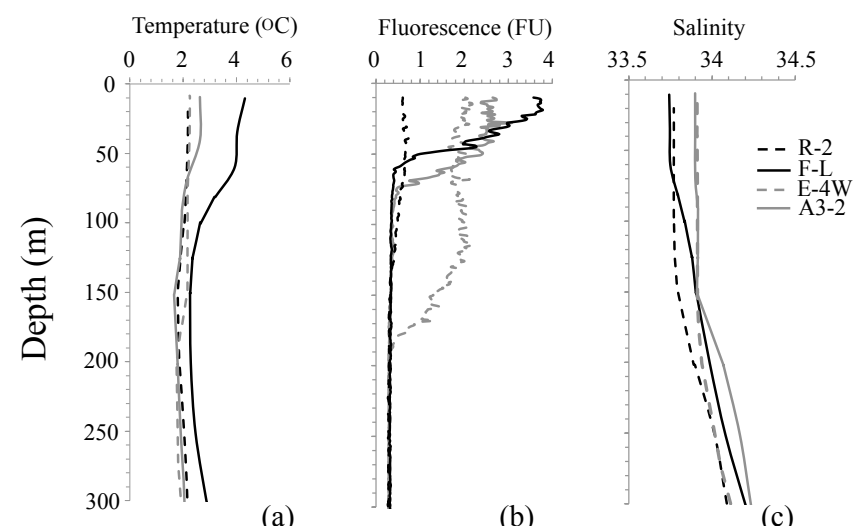

(b)

(c)

Figure 2. Profiles of temperature (a), $\mathrm{Chl} a$ as derived from in vivo fluorescence (b) and salinity (c) for each of the four sampling stations.

routines in the multivariate ecological research (PRIMER v.6) software package (Larke and Warwick, 2001). In order to identify inter-relationships between samples, BrayCurtis similarities were analysed by cluster analysis and nonmetric MDS on square-root sequence abundance. The similarity profile (SIMPROF) permutation test was conducted in PRIMER v.6 to establish the significance of dendrogram branches resulting from cluster analysis. Similarity percentage (SIMPER) analysis, also performed with PRIMER, was used to identify of the contribution of different OTUs to the observed similarity pattern.

\section{Results}

\subsection{Study site}

The hydrographic conditions during KEOPS2 are reported in detail in Park et al. (2014). The "historical" A3 station situated $\sim 500 \mathrm{~m}$ on the Kerguelen Plateau (Blain et al., 2007, 2008) was characterized by a deep mixed layer (ML) $(153 \pm 15 \mathrm{~m})$ (Table 1, Fig. 2). Stations F-L and E-4W revealed concentrations of 4.0 and $2.38 \mu \mathrm{g} \mathrm{L}-1 \mathrm{Chl} a$, respectively, constrained to shallow ML $(38 \pm 7 \mathrm{~m}$ and $61 \pm 11 \mathrm{~m}$, respectively; Table 1). The highest temperature was recorded in the $\mathrm{ML}$ of the F-L station $\left(4.2^{\circ} \mathrm{C}\right.$, Fig. 2$)$, indicating the influence of sub-Antarctic waters. The reference site (station R-2) in HNLC waters had low concentrations of Chl $a$ $\left(0.25 \pm 0.08 \mu \mathrm{g} \mathrm{L}{ }^{-1}\right)$, and a temperature of $2.1^{\circ} \mathrm{C}$ (Fig. 2) in the ML $(105 \pm 15 \mathrm{~m})$. The macronutrient concentrations in all 16 sampling points were high: $\sim 20-26 \mu \mathrm{M}$ for nitrate plus nitrite; $\sim 1-1.8 \mu \mathrm{M}$ for phosphate; $\sim 8-19 \mu \mathrm{M}$ for silicate; while dissolved iron was lower at the reference HNLC $\mathrm{R}-2$ station $(0.08 \mathrm{nM})$ relative to the iron-fertilized stations (0.16-0.22 nM; Table 2).

\subsection{Composition and distribution of protistan assemblages}

After quality filtering and normalization, 999 unique OTUs, clustering 50674 sequences (average length: $240 \mathrm{bp}$ ) were revealed for the 16 samples. The mean ratio of observed (Table 2$)$ to expected ( $S_{\text {chao1 }}$, Table 2 ) OTUs was $75 \pm 10 \%$ (mean \pm sd) for all depths and stations. The highest number of unique OTUs, considering all depths, was observed at the F-L station (711 OTUs), and the lowest at the E-4W station (387 OTUs), while A3-2 and the HNLC R-2 stations had similar number of OTUs (550 and 496, respectively). The Simpson index was relatively high, ranging from 0.76 (FL station in the ML) to 0.99 (HNLC, R-2 station at $300 \mathrm{~m}$ ). The Berger-Parker, indicating the relative abundance of the dominant OTU, was generally low, except at the F-L station, where it reached its highest value $(0.48$; Table 2$)$.

\subsubsection{High-level taxonomic groups}

The 999 OTUs were affiliated into 30 higher taxonomic groups distributed in all the samples (Table 3 ) and shown as pie charts for each of the four stations (Fig. 3). At all stations, Alveolata was the most diverse group (696 OTUs, mainly composed of MALV-II, Dinophyceae, MALV-I and Ciliophora). The iron-fertilized stations accounted for the highest percentages of Alveolata while the lowest percentage was observed at the HNLC station R-2 (Fig. 4). Stramenopiles were represented by 133 OTUs belonging to 10 higher taxonomic groups (Table 3). The most representative Stramenopile groups, in terms of OTUs number, were MAST, followed by Bacillariophyceae and Labyrinthulomycetes (Table 3). The relative abundance of sequences of Stramenopiles ranged between 8 and $29 \%$ in the mixed layer samples (Fig. 4). Radiolaria (belonging to Rhizaria) were present at all stations and were more abundant in the $300 \mathrm{~m}$ depth samples. Their relative abundance was particularly pronounced at station F-L, where they represented $55 \%$ of all sequences (Fig. 4). The fertilized stations were characterized by lower relative abundances of Haptophyta and Chlorophyta compared with the HNLC R-2 station (Fig. 4). Fungi were represented by relatively high OTU richness (28 OTUs; Table 3 ). They were found almost exclusively at the fertilized stations, when only three OTUs were detected at the HNLC R-2 station (Fig. 3).

Regarding lineages distribution according to depth, the proportions of phototrophic protists (e.g. Bacillariophyceae and Haptophyta) generally decreased below the ML. The relative contribution of MALV-I and MALV-II increased with depth, at all stations except at station F-L.

\subsubsection{Most abundant OTUs}

The most abundant 207 OTUs, representing $>1 \%$ of the sequences for each higher taxonomic group, accounted for $95 \%$ of the total sequences. 

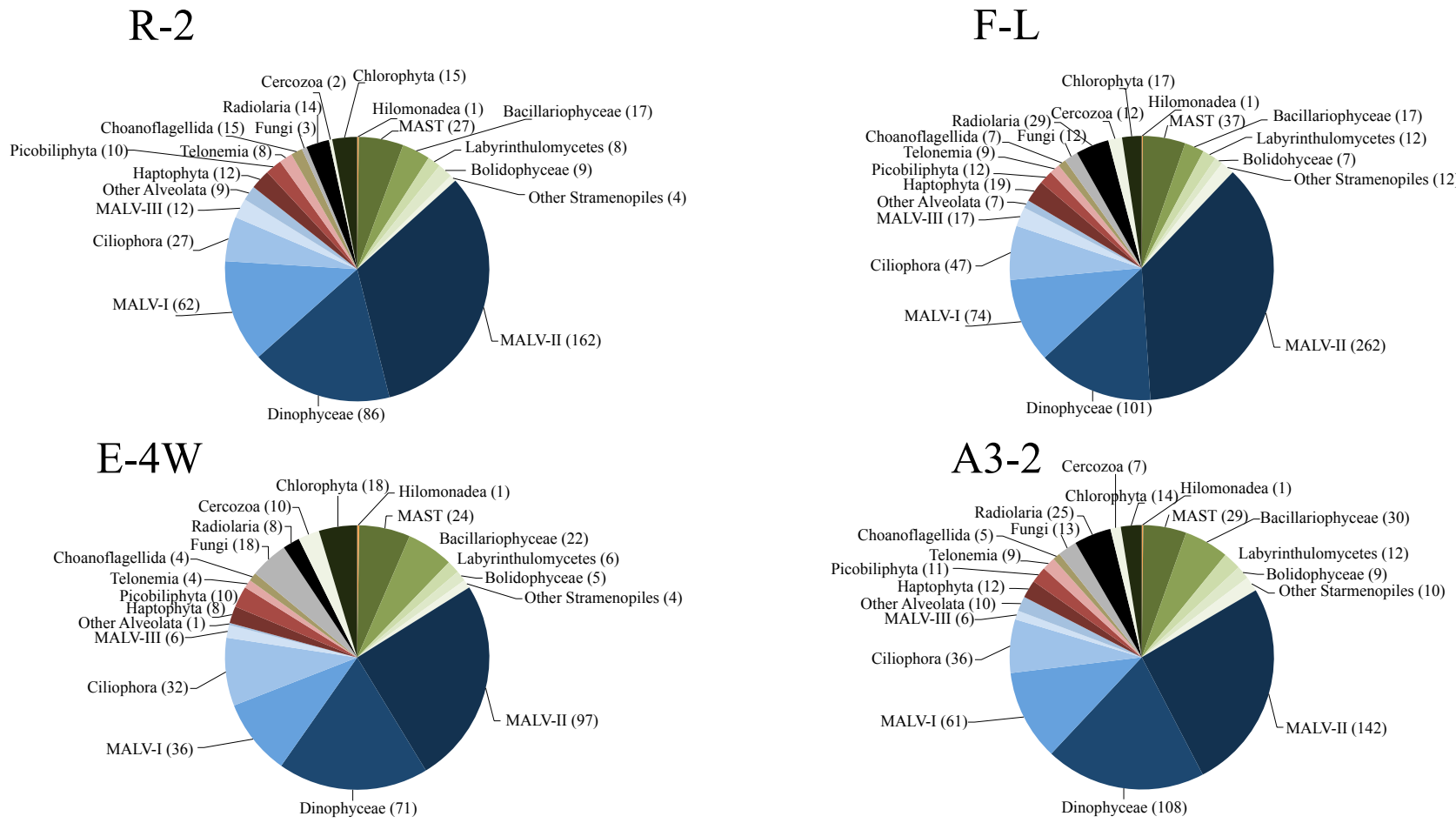

Figure 3. Overall diversity of major high-level taxonomic groups and number of OTUs indicated in parentheses at each station.

Table 2. Number of OTUs, the richness estimator $\left(S_{\text {chao1 }}\right)$, Simpson and Berger-Parker indices for each sample, number of sequences before and after removing metazoan and single singletons sequences.

\begin{tabular}{|c|c|c|c|c|c|c|c|}
\hline Station & $\begin{array}{l}\text { Depths } \\
(\mathrm{m})\end{array}$ & $\begin{array}{l}\text { No. } \\
\text { OTUs }\end{array}$ & $\begin{array}{c}\text { No. seqs } \\
\text { (before) }\end{array}$ & $\begin{array}{r}\text { No. seqs } \\
\quad \text { (after) }\end{array}$ & $S_{\text {chao1 }}$ & $\begin{array}{r}\text { Simpson } \\
(1-D)\end{array}$ & $\begin{array}{r}\text { Berger- } \\
\text { Parker }\end{array}$ \\
\hline \multirow[t]{4}{*}{ R-2 } & 20 & 157 & 5448 & 4714 & 198 & 0.95 & 0.18 \\
\hline & 60 & 170 & 6346 & 1522 & 218 & 0.95 & 0.16 \\
\hline & 150 & 233 & 4407 & 1562 & 390 & 0.97 & 0.13 \\
\hline & 300 & 282 & 1098 & 950 & 409 & 0.99 & 0.05 \\
\hline \multirow[t]{4}{*}{ F-L } & 20 & 186 & 5586 & 3028 & 253 & 0.76 & 0.48 \\
\hline & 65 & 508 & 7305 & 5730 & 663 & 0.98 & 0.08 \\
\hline & 180 & 265 & 7818 & 905 & 382 & 0.98 & 0.05 \\
\hline & 300 & 284 & 10205 & 2026 & 383 & 0.83 & 0.40 \\
\hline \multirow[t]{4}{*}{ E-4W } & 30 & 173 & 7151 & 6108 & 198 & 0.85 & 0.33 \\
\hline & 80 & 209 & 10977 & 6674 & 236 & 0.92 & 0.23 \\
\hline & 150 & 191 & 11989 & 5771 & 255 & 0.94 & 0.19 \\
\hline & 300 & 97 & 3178 & 242 & 174 & 0.97 & 0.08 \\
\hline \multirow[t]{4}{*}{ A3-2 } & 320 & 215 & 10666 & 1803 & 285 & 0.93 & 0.22 \\
\hline & 80 & 200 & 3866 & 2118 & 273 & 0.98 & 0.08 \\
\hline & 160 & 181 & 5986 & 2022 & 219 & 0.95 & 0.13 \\
\hline & 300 & 330 & 11590 & 5662 & 385 & 0.94 & 0.23 \\
\hline
\end{tabular}

The heterotrophic Gyrodinium spp. was the dominant Dinophyceae genus in all samples, while the small autotrophic Gymnodinium spp., also present in all samples, displayed higher relative abundance in the HNLC R-2 samples (Table 4). Among Ciliophora, the genus Strombidium was the most abundant, while different OTUs belonging to Tintinnid species (Choreotrichia) were detected at all stations. The 17 most representative MAST-related OTUs were distributed in eight clades, with a MAST-9 sp. prevailing at the surface F-L station (Table 4). 


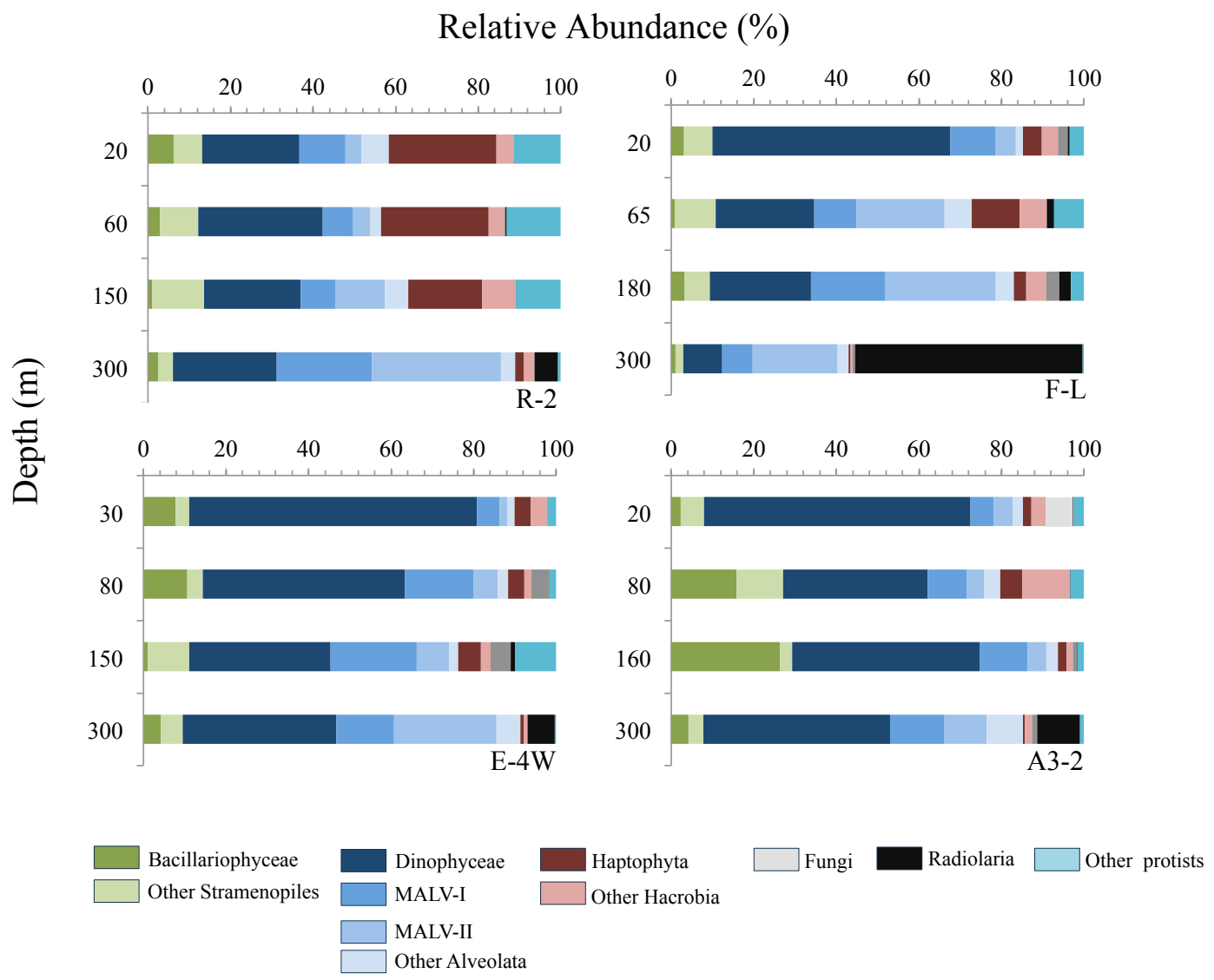

Figure 4. Relative abundance of major high-level taxonomic groups at each station and depth.

At the fertilized stations, Bacillariophyceae-related OTUs were dominated by small-sized species such as Planktoniella, Thalassiosira, and Minidiscus spp., while Pseudonitzschia was relatively abundant at the HNLC R-2 station (Table 4). Regarding the rest of the Stramenopiles, the photosynthetic picoalgae of the genus Bolidomonas prevailed at all stations. The non-photosythetic Labyrithulomycetes were more often found at the iron-fertilized stations, with the parasitic genus Oblongichytrium sp. being relatively more abundant at the E-4W and A3-2 stations (Table 4).

In all samples, the Haptophyta were dominated by Phaeocystis antarctica. Among Chlorophyta, Micromonas were better represented at the F-L and R-2 stations, while Pyramimonas spp. accounted for most of the Chlorophyta sequences at the A3-2 and E-4W stations. Choanoflagellates comprised eight OTUs, all belonging to the Stephanoecidae. Fungi were poorly represented at the HNLC R-2 station. Finally, Cercozoa were present at the iron-fertilized stations, but almost absent at the HNLC station R-2 (Table 4).

\subsection{Similarity of protistan assemblages}

Altogether, the stations shared 197 OTUs, with 40 OTUs specific to the fertilized stations (Fig. 5). The F-L station con- tained the highest number of exclusive OTUs (Fig. 5). The Bray-Curtis similarity analysis of 999 OTUs indicated four major clusters (Fig. 6a). The SIMPROF significance test indicated significant differences $(P<0.05)$ between these four groups and showed significant differences within the groups (i) to (iv) (Fig. 6a). The 2-D space nMDS visual representation, based on Bray-Curtis similarity analysis, highlighted two major clusters ("shallow" and "deep" samples). An overall low similarity ( $>15 \%$ ) was observed within each group (Fig. 6b). At a higher level of similarity (40-50\%), the clusters broke roughly into individual stations: HNLC (cluster i); A3-2 (cluster ii); and E-4W (cluster iii); while the F-L $20 \mathrm{~m}$ and $65 \mathrm{~m}$ samples clustered with E-4W and the HNLC stations, respectively (Fig. 6b). Within the "deep" assemblage (cluster iv), the similarity between samples was low, except for samples R $300 \mathrm{~m}$ and F-L $180 \mathrm{~m}$, which displayed $40 \%$ similarity (Fig. 6b). The SIMPER test highlighted the most relevant OTUs forming each cluster (Table 5). In the first cluster (i), the major contributor was Haptophyta (in particular P. antarctica), followed by Dinophyceae, and Chlorophyta. In the second cluster (ii), Dinophyceae contributed to $49.2 \%$ of the similarity, with G. spirale, having an important contribution together with 10 other Dinophyceae and Bacillariophyceae-related OTUs. In the third cluster (iii), 
Table 3. Higher-level taxonomic distribution of protistan OTUs defined at $97 \%$ sequence similarity.

\begin{tabular}{|c|c|c|}
\hline Supergroup & Taxonomic groups & OTUs \\
\hline \multirow[t]{9}{*}{ Alveolata } & MALV-II & 339 \\
\hline & Dinophyceae & 161 \\
\hline & MALV-I & 101 \\
\hline & Ciliophora & 60 \\
\hline & MALV-III & 21 \\
\hline & MALV-IV & 8 \\
\hline & Apicomplexa & 3 \\
\hline & MALV-V & 2 \\
\hline & Perkinsea & 1 \\
\hline \multirow[t]{10}{*}{ Stramenopiles } & MAST & 46 \\
\hline & Bacillariophyceae & 37 \\
\hline & Labyrinthulomycetes & 19 \\
\hline & Bolidophyceae & 13 \\
\hline & Pirsonia & 6 \\
\hline & Dictyochophyceae & 4 \\
\hline & Pelagophyceae & 3 \\
\hline & Hyphochrytriaceae & 2 \\
\hline & Oomyceta & 2 \\
\hline & Chrysophyceae & 1 \\
\hline \multirow{5}{*}{ Hacrobia } & Haptophyta & 20 \\
\hline & Picobiliphyta & 15 \\
\hline & Telonemia & 12 \\
\hline & Centroheliozoa & 2 \\
\hline & Cryptophyta & 1 \\
\hline \multirow[t]{2}{*}{ Opisthokonta } & Fungi & 28 \\
\hline & Choanoflagellida & 10 \\
\hline \multirow[t]{2}{*}{ Rhizaria } & Radiolaria & 35 \\
\hline & Cercozoa & 17 \\
\hline Archaeplastida & Chlorophyta & 29 \\
\hline Apusozoa & Hilomonadea & 1 \\
\hline
\end{tabular}

Dinophyceae also prevailed (58.6\% of the similarity), with two OTUs affiliated to G. spirale, where G. rubrum was the most important. Finally, the last cluster (iv), representing the "deep" samples, was characterized by MALV-II and Radiolaria.

\section{Discussion}

\subsection{Overview of the commonly occurring taxa according to tag pyrosequencing}

This is the first broad study of protist community composition in the natural iron-fertilized Kerguelen area of the Southern Ocean. The overall taxonomic diversity of protists recovered included 999 OTUs, belonging to 30 high-level taxonomic groups. A total of 207 OTUs were classified as "abundant" (each representing $\geq 1 \%$ of sequences in their higher

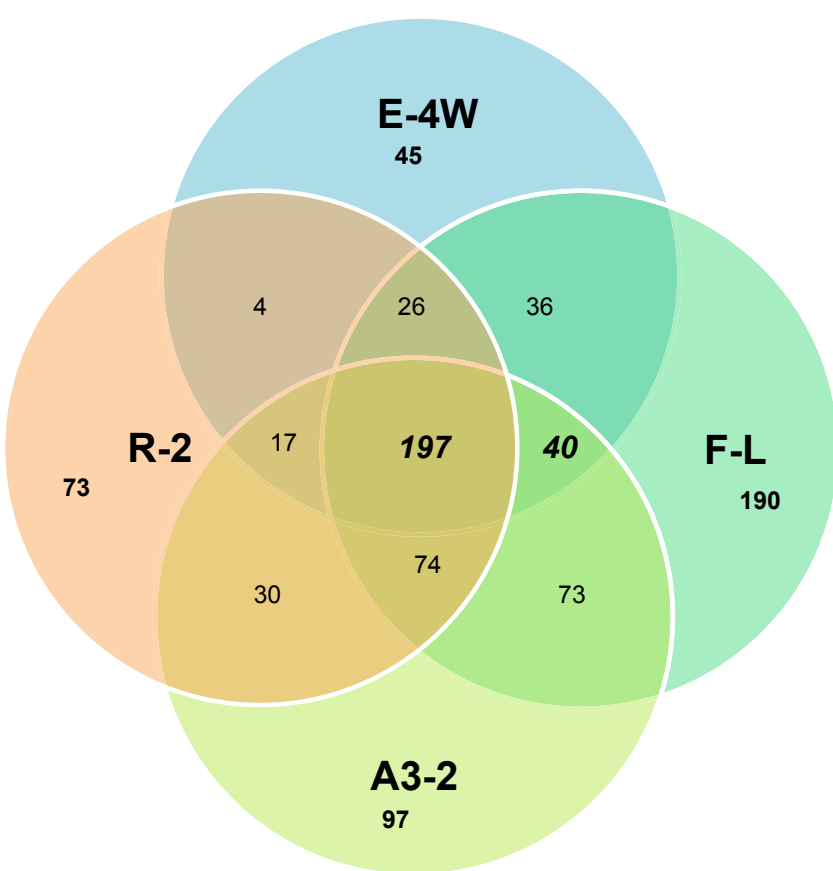

Figure 5. Venn diagrams representing the number of OTUs shared between the different stations.

taxonomic group) (Table 4); the most frequent OTUs belonged to Alveolata, followed by Stramenopiles, then Hacrobia (Table 3).

\subsubsection{Phytoplankton}

Although the tag pyrosequencing of the 18S rRNA gene has become a routine method in marine microbial diversity studies, it is itself subjected to several limitations, including, DNA extraction and PCR-related biases, chimera formation, and primer non-universality (e.g. Prokopowich et al., 2003; Ki and Han, 2005; Zhu et al., 2005; Edgcomb et al., 2011). Although it has been established that Bacillariophyceae respond to iron fertilization by rapidly forming extensive blooms (for a review see Quéguiner, 2013), concerning this study only 11 out of the 38 OTUs affiliated to Bacillariophyceae were found in common with the 52 diatom taxa morphologically identified in the Kerguelen area during the KEOPS 1 cruise at the end of the bloom period (Armand et al., 2008).

According to KEOPS2's microscopical observations and pigment analysis data, Bacillariophyceae dominated the phytoplankton community in the blooms (Sackett et al., 2014; Lasbleiz et al., 2014). In particular, Fragilariopsis kerguelensis, Pseudonitzschia spp., Eucampia antarctica, and Chaetoceros spp. were found to be the four dominant diatom taxa, via microscopy (Sackett et al., this volume). However, while Pseudonitzschia-, Eucampia- and Chaetoceros-related OTUs represented $14 \%$ of the Bacillariophyceae-related sequences, no Fragilariopsis-related OTUs were detected. 
Table 4. Colour-coded heat-map table of the major taxonomic groups ( $>10$ OTUs) (see Table 3). The 207 OTUs presented here accounted for $95 \%$ of the total sequences and represented $>1 \%$ of sequences in each taxonomic group. The colours represent the relative abundance of each OTU within each sample. White boxes indicate absence. Black contours indicate the 17 OTUs found only at one station.

\begin{tabular}{|c|c|c|c|c|c|c|c|c|c|c|c|c|c|c|c|c|c|c|}
\hline$A b$ & & $0.1-10 \%$ & 11 to 2 & & & & $26 \mathrm{t}$ & $40 \%$ & & & & to $60 \%$ & & & & $60 \%$ & & \\
\hline & OTUs & Taxonomic affiliation & Identity & $\mathrm{R}-2$ & & & & F-L & & & & $E-4 W$ & & & & A3-2 & & \\
\hline & & & & Е్ & $\begin{array}{l}\varepsilon \\
\text { ¿ } \\
\end{array}$ & ह్ & छ్ & క్ & हૃ & 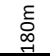 & है & క్ & క్ & 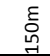 & छ్ & క్ & ह్ & $\underset{్}{E}$ \\
\hline & Otu1211 & Dino-Group-II-Clade0-and1 sp. & 98 & & $\overline{6.3}$ & 1.8 & 1.7 & 11.9 & 7.6 & $\overline{17.6}$ & 8.9 & & 1.8 & 4.1 & 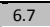 & 1.1 & $\overline{5.6}$ & 2.5 \\
\hline & Otu1633 & Dino-Group-II-Clade-5 sp. & 90 & 36.8 & 6.3 & 1.6 & & & & 1.7 & 1.2 & 16.5 & 19.4 & 9.3 & & 3.8 & 1.1 & 28.9 \\
\hline & Otu1419 & Dino-Group-II-Clade0-and1 sp. & 100 & 11.4 & 14.3 & 8.2 & 0.3 & 1.4 & 6.0 & 4.2 & & 4.3 & 18.8 & 4.6 & & 2.5 & & 2.5 \\
\hline & Otu1179 & Dino-Group-II-Clade6 sp. & 100 & 2.7 & & 2.7 & 1.3 & 4.2 & 2.9 & 2.9 & 0.8 & 3.5 & 9.2 & 2.8 & 1.7 & 1.3 & 4.5 & 6.2 \\
\hline & Otu1613 & Dino-Group-II-Clade-7 sp. & 100 & & & & 5.4 & 0.7 & 12.9 & 4.6 & 1.9 & & & & 3.3 & & & \\
\hline & Otu0595 & Dino-Group-II-Clade-6 sp. & 100 & & 1.6 & 7.3 & 0.7 & 4.2 & 3.8 & 1.3 & 1.4 & 2.7 & & 1.6 & & 5.6 & 15.7 & 1.8 \\
\hline & Otu1332 & Dino-Group-II-Clade3 sp. & 96 & 0.5 & 9.5 & 8.2 & & 6.3 & 2.3 & 0.8 & & 0.9 & 15.2 & 3.9 & & & 2.2 & \\
\hline & Otu1116 & Dino-Group-II-Clade0-and1 sp. & 100 & & & 2.2 & 9.8 & & 0.7 & 4.6 & 0.8 & & & 1.9 & 15.0 & & 1.1 & 1.2 \\
\hline & Otu1183 & Dino-Group-II-Clade-7 sp. & 100 & & & & 1.3 & & & 0.4 & 0.8 & & & & 23.3 & & & \\
\hline & Otu1743 & Dino-Group-II-Clade sp. & 92 & 7.3 & 1.6 & 1.6 & 0.7 & & 1.7 & 0.4 & & & & 5.6 & & 3.8 & & \\
\hline & Otu1926 & Dino-Group-II-Clade-6 sp. & 100 & & 1.6 & 0.5 & 0.3 & & 2.3 & 6.7 & 1.9 & & & 0.4 & & 2.5 & & \\
\hline & Otu1455 & Dino-Group-II-Clade0-and1 sp. & 100 & & & 3.2 & & 0.7 & 1.9 & 1.3 & & 0.9 & 3.9 & 1.9 & & & 5.6 & 4.8 \\
\hline & Otu1947 & Dino-Group-II-Clade-20 sp. & 94 & & 3.2 & 0.5 & 0.7 & 0.7 & 3.4 & 1.7 & 0.9 & & & & & & & 2.5 \\
\hline & Otu1663 & Dino-Group-II-Clade-7 sp. & 100 & & & 1.8 & 3.7 & & 0.5 & 0.8 & 3.7 & & & & 11.7 & & & \\
\hline & Otu1771 & Dino-Group-II-Clade-7 sp. & 95 & 0.5 & & 3.2 & & 1.4 & 2.0 & & & 6.9 & & 2.9 & & & & \\
\hline & Otu1827 & Dino-Group-II-Clade0-and1 sp. & 97 & & & & 0.3 & & 2.4 & 0.8 & & & 1.5 & 1.4 & & 8.9 & 5.6 & 2.5 \\
\hline ฉ & Otu1584 & Dino-Group-II-Clade-7 sp. & 99 & & & & 2.7 & & 1.5 & 2.5 & 0.2 & & & 0.4 & 1.7 & 7.6 & & 6.2 \\
\hline ڤ్ & Otu0567 & Dino-Group-II sp. & 93 & & & 3.8 & & & 0.6 & 0.4 & & 1.7 & 5.5 & 1.9 & & & 1.1 & \\
\hline $\bar{s}$ & Otu1874 & Dino-Group-II-Clade-30 sp. & 98 & & & & 0.7 & 13.9 & 1.1 & & 0.2 & 1.7 & 0.8 & 0.3 & & & 3.4 & \\
\hline 3 & Otu0241 & Dino-Group-II sp. & 100 & & & & & & 0.8 & & 0.2 & & 0.8 & 8.3 & & & & \\
\hline$\sum_{\Sigma}^{a}$ & Otu1468 & Dino-Group-II-Clade-7 sp. & 87 & & & & & 0.7 & 3.6 & & & & & & & & & \\
\hline & Otu1951 & Gyrodinium spirale & 100 & 27.6 & 13.8 & 2.6 & 22.1 & \begin{tabular}{|l|}
83.4 \\
\end{tabular} & 5.0 & 5.4 & 2.1 & 47.6 & 47.7 & 9.9 & & 34.7 & 18.0 & 28.1 \\
\hline & Otu1914 & Gyrodinium rubrum & 96 & 1.4 & 0.2 & 0.8 & & 1.3 & 12.2 & 20.0 & & 25.0 & 31.8 & 58.0 & & 5.8 & 6.1 & 15.0 \\
\hline & Otu1777 & Pentapharsodinium sp. & 97 & 0.9 & 3.7 & 3.3 & & & 0.7 & & 10.0 & 0.8 & & 0.7 & 2.2 & 2.1 & 0.7 & 1.8 \\
\hline & Otu1967 & Gymnodinium sp. & 99 & 17.3 & 25.9 & 28.8 & 11.6 & 2.6 & 11.4 & 12.2 & 8.2 & 4.3 & 1.9 & 8.6 & 7.8 & 3.3 & 5.7 & 3.7 \\
\hline & Otu1898 & Karlodinium micrum & 97 & 0.6 & 2.0 & 1.6 & 0.4 & 1.5 & 8.4 & 2.3 & 3.3 & 7.4 & 4.9 & 2.6 & 1.0 & 1.7 & 1.7 & 5.8 \\
\hline & Otu1016 & Gyrodinium sp. & 98 & 4.9 & 5.3 & 2.2 & 3.4 & 0.7 & 11.6 & 8.6 & 1.7 & 4.0 & 2.4 & 0.8 & 22.2 & 4.5 & 4.2 & 4.3 \\
\hline ฉ & Otu1770 & Warnowia sp. & 96 & 2.9 & 1.5 & 2.7 & 4.7 & 1.3 & 2.5 & 3.6 & 21.9 & 1.8 & 1.6 & 3.8 & 12.2 & 9.3 & 4.5 & 3.2 \\
\hline ஜे & Otu1763 & Dinophyceae sp. & 100 & 18.2 & 14.5 & 2.7 & 7.2 & 0.7 & 0.7 & 1.4 & 1.6 & 0.4 & 1.1 & 2.7 & 6.7 & 3.5 & 12.2 & 4.8 \\
\hline$\overline{0}$ & Otu1808 & Warnowia sp. & 97 & 1.4 & 9.2 & 1.9 & 1.7 & 0.3 & 5.0 & 4.7 & 4.3 & 2.5 & 0.9 & 1.8 & & 4.5 & 4.6 & 4.5 \\
\hline đ్ & Otu1744 & Dinophyceae sp. & 100 & 1.3 & 4.8 & 1.6 & 0.4 & 0.2 & 5.6 & 2.7 & 3.3 & 0.8 & 1.0 & 1.7 & 2.2 & 0.3 & 1.4 & 2.3 \\
\hline$\stackrel{\vec{e}}{\vec{t}}$ & Otu1722 & Gymnodinium sp. & 96 & 0.9 & 1.8 & 2.2 & 0.4 & 0.6 & 5.5 & 2.3 & 1.7 & 1.2 & 1.2 & & 2.2 & 1.9 & 2.9 & 1.1 \\
\hline 응 & Otu1953 & Pentapharsodinium tyrrhenicum & 98 & 0.5 & 2.4 & 3.8 & 6.0 & 0.6 & 3.2 & 3.6 & 1.6 & 0.6 & 1.1 & 0.5 & 4.4 & 1.7 & 1.8 & 2.0 \\
\hline : & Otu1793 & Katodinium rotundatum & 95 & 1.3 & 2.0 & 2.0 & 1.3 & 2.6 & 3.4 & 0.9 & 1.6 & 0.8 & 0.6 & 1.9 & & 1.9 & 0.7 & 0.7 \\
\hline & Otu1912 & Dino-Group-I-Clade-1 sp. & 100 & 4.4 & 7.3 & 9.2 & 6.4 & 5.2 & 11.9 & 8.7 & 0.7 & 24.8 & 31.9 & 28.3 & 2.9 & 16.2 & 16.3 & 11.4 \\
\hline & Otu1790 & Dino-Group-I-Clade-4 sp. & 100 & 52.9 & 22.7 & 6.2 & 7.4 & 1.2 & 5.7 & 1.2 & 2.6 & 12.8 & 13.8 & 39.8 & 2.9 & 2.2 & & 7.9 \\
\hline & Otu1653 & Dino-Group-I-Clade-1 sp. & 100 & 2.6 & 25.5 & 12.4 & 2.9 & 0.7 & 8.3 & 19.3 & 16.3 & 3.8 & 24.6 & 13.7 & 17.6 & 19.2 & 14.8 & 22.3 \\
\hline & Otu1285 & Dino-Group-I-Clade-1 sp. & 100 & 11.4 & 11.8 & 9.8 & 1.8 & 59.2 & 8.6 & 13.7 & & 15.2 & 22.5 & 2.5 & 5.9 & 11.1 & 3.6 & 25.3 \\
\hline & Otu1393 & Dino-Group-I-Clade-1 sp. & 97 & 1.6 & 10.0 & 6.2 & 0.5 & 1.5 & 22.7 & 3.7 & & 2.1 & 0.8 & 3.0 & & 6.7 & 2.6 & 0.4 \\
\hline ळ & Otu1292 & Dino-Group-I-Clade-5 sp. & 100 & & 3.6 & 3.8 & 1.5 & & 0.3 & 2.5 & 6.5 & & 0.7 & 1.7 & 5.9 & 26.3 & 48.0 & 16.2 \\
\hline స్ & Otu0979 & Dino-Group-I-Clade-4 sp. & 100 & 3.9 & 2.7 & 3.8 & 0.5 & 15.6 & 3.0 & 2.5 & 2.6 & 3.9 & 2.6 & 3.2 & 5.9 & & & 1.7 \\
\hline$\overline{1}$ & Otu1753 & Dino-Group-I-Clade-1 sp. & 96 & 2.3 & 6.4 & 13.5 & & 3.3 & 14.2 & 6.2 & & 3.2 & 2.9 & 0.3 & & 4.4 & 7.1 & \\
\hline 之্ & Otu0518 & Dino-Group-I-Clade-2 sp. & 98 & & & 1.5 & 0.5 & & 2.0 & 1.2 & 0.7 & & 0.2 & 7.2 & & 1.1 & 2.5 & 1.7 \\
\hline$\Sigma$ & Otu1920 & Dino-Group-I-Clade-4 sp. & 98 & 6.4 & & 1.5 & 0.9 & 1.0 & 2.3 & 4.3 & 1.4 & & & 0.2 & 2.9 & 1.1 & & 0.9 \\
\hline & Otu1799 & Strombidium biarmatum & 100 & 26.7 & 25.9 & 28.6 & 21.7 & 26.7 & 34.4 & 25.9 & 2.4 & 34.4 & 44.0 & 6.4 & 15.4 & 2.0 & 32.9 & 13.3 \\
\hline & Otu1692 & Strombidiidae sp. & 100 & 21.5 & 18.5 & & & & 2.2 & 11.1 & 14.3 & & & & 15.4 & & 21.4 & 1.9 \\
\hline & Otu1310 & Strombidiidae sp. & 100 & 0.8 & & 2.6 & & 36.7 & 12.4 & & & 15.6 & 1.7 & 43.6 & & 27.5 & 18.6 & 15.9 \\
\hline & Otu1672 & Choreotrichia sp. & 98 & & & 1.6 & 13.4 & & 1.9 & 3.7 & 2.4 & & 0.7 & 14.1 & 23.8 & 5.0 & 2.9 & 13.3 \\
\hline & Otu0922 & Pelagostrobilidium neptuni & 99 & 14.4 & & 1.6 & & 6.7 & 0.7 & & & 4.2 & 2.0 & & & & & 5.7 \\
\hline & Otu1845 & Choreotrichia sp. & 95 & 2.3 & & 1.6 & 4.3 & 3.3 & 8.3 & & & & 0.7 & & & 5.0 & & 28.3 \\
\hline & Otu1773 & Laboea strobila & 97 & 4.3 & 29.6 & & & 3.3 & 0.4 & & & 9.4 & 11.3 & & & & & \\
\hline & Otu0371 & Cymatocylis calyciformis & 100 & 1.6 & & & & 3.3 & & & & & 12.0 & & & & 1.4 & \\
\hline & Otu1018 & Strobilidium caudatum & 96 & 1.1 & 3.7 & & & & 1.5 & 7.5 & & 3.1 & & 1.3 & & 2.5 & 5.7 & \\
\hline & Otu1467 & Pseudotontonia sp & 100 & 2.7 & 11.1 & 3.2 & & 3.3 & & & 2.4 & 14.6 & 2.0 & & & 12.5 & 2.9 & 1.9 \\
\hline & Otu0336 & Collinia beringensis & 98 & & & & & & & & 7.1 & & & 28.3 & & & & \\
\hline & Otu1053 & Mesodiniidae sp. & 98 & & & 1.6 & 34.8 & & 1.9 & 3.7 & 4.0 & & & & 7.7 & & & \\
\hline & Otu0145 & Pseudotontonia simplicidens & 100 & 2.7 & & & & & 2.2 & & & 6.3 & 4.7 & & & 2.5 & & \\
\hline & Otu1601 & Scuticociliatia sp. & 97 & & & & 8.7 & & 1.9 & & 7.1 & & & & & 2.5 & & \\
\hline 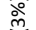 & Otu1320 & Choreotrichia sp. & 93 & & & & & & 4.4 & 3.7 & & 2.8 & 0.7 & & & 2.5 & 2.9 & 1.9 \\
\hline $\int_{0}^{\infty}$ & Otu0516 & Mesodiniidae sp. & 89 & & & 1.6 & & & 2.6 & & & & 0.7 & & & & & \\
\hline ఏ & Otu1317 & Colpodea sp. & 87 & & & 7.9 & & & 2.2 & 3.7 & & 3.1 & 0.7 & & & & & \\
\hline 응 & Otu1614 & Strombidiidae sp. & 99 & & & & & & 4.4 & & 2.4 & & & & & & & 5.7 \\
\hline$\overline{\bar{z}}$ & Otu1322 & Strombidium basimorphum & 98 & & & & & & 5.2 & 3.7 & & & & & & 2.5 & & \\
\hline & Otu1426 & Dino-Group-III sp. & 100 & 20.0 & 81.8 & 27.3 & 50.0 & & 21.5 & 33.3 & & 6.7 & & & & & 72.7 & \\
\hline & Otu1030 & Dino-Group-III sp. & 95 & 26.0 & & 18.2 & & & 13.9 & 11.1 & & & 36.4 & 100 & & 20.0 & & 100 \\
\hline & Otu1731 & Dino-Group-III sp. & 95 & 10.0 & 18.2 & 18.2 & & 5.6 & 22.8 & 11.1 & & 13.3 & & & & & & \\
\hline & Otu1911 & Dino-Group-III sp. & 95 & 30.0 & & 9.1 & & 5.6 & 2.5 & 11.1 & & & & & & & & \\
\hline & Otu0008 & Dino-Group-III sp. & 93 & & & & & & 1.3 & & & 66.7 & & & & 60.0 & 27.3 & \\
\hline & Otu1429 & Dino-Group-III sp. & 93 & & & & & 83.3 & & & & & & & & & & \\
\hline & Otu1958 & Dino-Group-III sp. & 99 & 2.0 & & 4.5 & & & 15.2 & & & & & & & & & \\
\hline & Otu1093 & Dino-Group-III sp. & 89 & & & 9.1 & & 5.6 & 7.6 & 11.1 & & 13.3 & & & & & & \\
\hline & Otu1664 & Dino-Group-III sp. & 89 & 12.0 & & & & & 1.3 & 11.1 & & & & & & & & \\
\hline ํํำ & Otu0882 & Dino-Group-III sp. & 89 & & & & & & & & & & 63.6 & & & & & \\
\hline$\subseteq$ & Otu1609 & Dino-Group-III sp. & 94 & & & & & & & & 100.0 & & & & & & & \\
\hline$\equiv$ & Otu1255 & Dino-Group-III sp. & 97 & & & & & & 3.8 & & & & & & & 20.0 & & \\
\hline 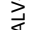 & Otu0398 & Dino-Group-III sp. & 92 & & & & & & & & & & & & & & & \\
\hline$\sum$ & Otu1277 & Dino-Group-III sp. & 95 & & & & & & 3.8 & & & & & & & & & \\
\hline
\end{tabular}


Table 4. Continued.

\begin{tabular}{|c|c|c|c|c|c|c|c|c|c|c|c|c|c|}
\hline & $\begin{array}{l}\text { Otu1762 } \\
\text { Otu1923 }\end{array}$ & $\begin{array}{l}\text { MAST-1B sp. } \\
\text { MASTC sp. }\end{array}$ & $\begin{array}{l}100 \\
100\end{array}$ & \begin{tabular}{|l|}
2.4 \\
17.2
\end{tabular} & $\begin{array}{l}15.3 \\
2.3\end{array}$ & $\begin{array}{l}27.7 \\
16.8\end{array}$ & $\begin{array}{l}1.0 \\
15.0\end{array}$ & \begin{tabular}{|l|}
24.3 \\
6.4
\end{tabular} & $\begin{array}{l}25.7 \\
1.7\end{array}$ & $\begin{array}{l}23.8 \\
7.7\end{array}$ & $\begin{array}{l}9.5 \\
9.5\end{array}$ & \begin{tabular}{|l|}
6.0 \\
12.0
\end{tabular} & $\begin{array}{l}9.2 \\
6.2\end{array}$ \\
\hline & Otu0923 & MAST-8 sp. & 100 & 1.3 & 3.4 & 19.8 & & 2.3 & 6.0 & 2.6 & & 3.6 & 3.8 \\
\hline & Otu1031 & MAST-1C sp. & 100 & 1.3 & 8.5 & 1.0 & 1.0 & 2.3 & 9.1 & 7.7 & & & 4.6 \\
\hline & Otu0641 & MAST-3 sp. & 84 & & & & & 4.6 & 1.0 & & & 13.8 & 1.0 \\
\hline & Otu1618 & MAST-9 sp. & 99 & 2.0 & & & & & 0.7 & 7.7 & 4.8 & & 18.5 \\
\hline & Otu0656 & MAST-2 sp. & 100 & 12.5 & 6.8 & 1.0 & & 1.2 & 2.4 & & 9.5 & 28.1 & 19.2 \\
\hline & Otu1009 & MAST-1A sp. & 100 & 5.9 & 1.2 & 7.9 & 15.0 & 2.3 & 8.2 & 2.6 & & 5.4 & 6.2 \\
\hline & Otu1638 & MAST-9 sp. & 97 & & & & & 42.8 & 0.5 & 5.1 & & & 6.9 \\
\hline & Otu1205 & MAST-7 sp. & 100 & 0.7 & 3.4 & 5.9 & & 1.2 & 3.6 & 5.1 & 4.8 & 4.2 & 0.8 \\
\hline & Otu1908 & MAST-3 sp. & 95 & 2.0 & 5.8 & 1.0 & & & 5.5 & 5.1 & & 9.0 & 3.8 \\
\hline & Otu1788 & MAST-3 sp. & 96 & 3.9 & 11.9 & 4.0 & & & 1.2 & 5.1 & & 3.6 & 5.4 \\
\hline & Otu1235 & MAST-7 sp. & 98 & 1.3 & & & & 4.6 & 6.7 & & & 1.2 & 4.6 \\
\hline a & Otu0973 & MAST-1A sp. & 100 & 17.2 & 1.7 & & & 1.7 & 2.9 & & & 3.6 & \\
\hline$\stackrel{8}{ \pm}$ & Otu1208 & MAST-7 sp. & 96 & 3.9 & 5.8 & 10.0 & & 1.2 & 3.4 & & & 3.6 & \\
\hline 5 & Otu1567 & MAST-3 sp. & 95 & 9.9 & & & 5.0 & 1.2 & 1.0 & 5.1 & 4.8 & 3.0 & \\
\hline$\sum$ & Otu1546 & MAST-3 sp. & 87 & & & & & & 1.7 & & 4.8 & 0.6 & 0.8 \\
\hline & Otu1447 & Planktoniella sol & 100 & 3.9 & 25.6 & 11.8 & 22.7 & 42.6 & 18.5 & 24.1 & 57.1 & 6.3 & 29.8 \\
\hline & Otu1564 & Coscinodiscus trioculatus & 100 & & 2.3 & 5.9 & & 47.9 & 24.7 & 2.7 & 19.5 & 11.4 & 16.9 \\
\hline & Otu0904 & Coscinodiscus sp. & 95 & & & & & & & & & 15.4 & 16.8 \\
\hline & Otu0581 & Rhizosolenia styliformis & 100 & & & & & 2.1 & & & 4.8 & 4.8 & 0.4 \\
\hline & Otu1786 & Pseudo-nitzschia pungens & 100 & 52.6 & 37.3 & & 22.7 & & 1.9 & & & & 1.7 \\
\hline 8̊ & Otu1293 & Corethron pennatum & 95 & 8.3 & 9.3 & 5.9 & & & 1.9 & & & 2.8 & 18.0 \\
\hline$\frac{0}{0}$ & Otu0978 & Thalassiosira delicatula & 100 & & & & & 1.6 & & 3.4 & & 0.4 & 0.3 \\
\hline$\underset{\widetilde{J}}{\pi}$ & Otu1787 & Actinocyclus actinochilus & 99 & 14.9 & 14.0 & 35.3 & 13.6 & & 14.8 & 13.8 & & & 1.9 \\
\hline$\stackrel{U}{\Xi}$ & Otu1372 & Pseudo-nitzschia multiseries & 99 & 16.3 & 7.0 & & 13.6 & & 1.9 & & & 0.6 & \\
\hline 흥 & Otu0005 & Thalassionema nitzschioides & 100 & & & & & & & & & & 1.3 \\
\hline 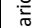 & Otu0184 & Guinardia flaccida & 92 & & & & & & & & & & 3.6 \\
\hline 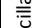 & Otu1536 & Eucampia antarctica & 100 & & & 29.4 & 22.7 & & 1.9 & & 4.8 & & \\
\hline లై & Otu0756 & Porosira pseudodenticulata & 100 & & & & & & 11.1 & 31.0 & 14.3 & & \\
\hline$\frac{5}{2}$ & Otu0727 & Oblongichytrium sp. & 90 & & & 14.3 & & 18.2 & 6.0 & 11.1 & 20.0 & & 72.1 \\
\hline$\hat{0}$ & Otu0512 & Labyrinthulaceae sp. & 92 & & 16.7 & & & 45.5 & 30.0 & 11.1 & 26.7 & & 8.2 \\
\hline$\frac{3}{n}$ & Otu0042 & Labyrinthulaceae sp. & 100 & & & & 50.0 & & 26.0 & 55.6 & 13.3 & & \\
\hline \pm & Otu0984 & Labyrinthulaceae sp. & 88 & & 33.3 & 85.7 & & 4.5 & 16.0 & & & 100.0 & 3.3 \\
\hline$\Xi$ & Otu1330 & Oblongichytrium sp. & 91 & & & & & & & & & & 14.8 \\
\hline$\underline{\Xi}$ & Otu0193 & Oblongichytrium sp. & 92 & & & & & & 16.0 & 11.1 & 13.3 & & \\
\hline$\frac{0}{3}$ & Otu1747 & Labyrinthulaceae sp. & 90 & & & & 25.0 & & & & 20.0 & & \\
\hline 言 & Otu0468 & Oblongichytrium sp. & 91 & & & & & 27.3 & & & & & \\
\hline$\sum_{2}^{2}$ & Otu0253 & Labyrinthulaceae $s p$. & 94 & & & & & & & & & & 1.6 \\
\hline$\frac{0}{3}$ & Otu0717 & Oblongichytrium sp. & 90 & & 16.7 & & & & & 11.1 & 6.7 & & \\
\hline$\overline{2}$ & Otu1213 & Bolidophyceae sp. & 99 & 30.8 & 33.3 & 12.5 & & & 25.0 & 33.3 & & 80.8 & 68.2 \\
\hline$\vec{m}$ & Otu1903 & Bolidomonas mediterranea & 90 & 30.8 & 13.3 & 87.5 & & & 52.8 & & & 3.8 & 29.5 \\
\hline$\stackrel{m}{0}$ & Otu0192 & Bolidophyceae sp. & 93 & 28.2 & 20.0 & & & & & & & & \\
\hline ฮँ & Otu1883 & Bolidophyceae sp. & 90 & & & & 66.7 & 80.0 & 5.6 & 66.7 & & & \\
\hline$\stackrel{u}{\simeq}$ & Otu0147 & Bolidomonas mediterranea & 95 & 10.3 & & & & & 2.8 & & & & \\
\hline 응 & Otu0624 & Bolidomonas pacifica & 97 & & 20.0 & & & 20.0 & 8.3 & & & & \\
\hline$\stackrel{0}{0}$ & Otu0016 & Bolidophyceae sp. & 88 & & & & & & & & & 15.4 & \\
\hline$\overline{0}$ & Otu1713 & Bolidomonas mediterranea & 90 & & 6.7 & & & & 2.8 & & & & 2.3 \\
\hline & Otu1782 & Phaeocystis antarctica & 100 & 67.3 & 62.8 & 71.2 & 58.8 & 78.7 & 66.6 & 53.3 & & 69.7 & 65.2 \\
\hline & Otu1907 & Chrysochromulina strobilus & 100 & 5.2 & 1.7 & 9.3 & 11.8 & 11.3 & 11.7 & 13.3 & 14.3 & 2.9 & 26.2 \\
\hline & Otu1884 & Chrysochromulina sp. & 97 & 11.6 & 19.8 & 17.4 & 5.9 & 9.6 & 1.8 & & $\mid 14.3$ & 6.0 & 3.3 \\
\hline$\frac{1}{\circ}$ & Otu1778 & Gephyrocapsa oceanica & 100 & 13.1 & 3.5 & & 17.6 & & 3.0 & 3.3 & & & \\
\hline 을 & Otu1026 & E anthemachrysis gayraliae & 93 & 0.2 & 0.8 & 1.7 & & & 2.2 & & & 3.4 & 5.3 \\
\hline 容 & Otu1774 & Chrysochromulina hirta & 100 & 1.0 & 1.2 & & 5.9 & 0.7 & 1.2 & & 57.1 & & \\
\hline & Otu1423 & Picobiliphyta sp. & 94 & 57.0 & 16.7 & 33.3 & 4.5 & 6.8 & 25.6 & 32.5 & 50.0 & 29.6 & 11.8 \\
\hline & Otu1067 & Picobiliphyta sp. & 100 & & 7.1 & 21.6 & & 43.6 & 17.9 & 17.5 & 16.7 & 18.5 & 26.9 \\
\hline อे & Otu1965 & Picobiliphyta sp. & 99 & 4.7 & 14.3 & 17.1 & 40.9 & 18.8 & 8.2 & 12.5 & & 12.0 & 14.0 \\
\hline$\vec{m}$ & Otu1899 & Picobiliphyta sp. & 99 & & 2.4 & 2.7 & & $\mid 17.1$ & 21.2 & 5.0 & & 15.0 & 12.9 \\
\hline $\bar{J}$ & Otu1387 & Picobiliphyta sp. & 96 & 17.4 & 21.4 & 9.9 & 22.7 & 0.9 & 5.3 & 12.5 & & 6.9 & 22.6 \\
\hline ț & Otu1025 & Picobiliphyta sp. & 92 & 20.9 & 28.6 & 6.3 & 4.5 & 0.9 & 6.2 & & & 5.2 & 4.3 \\
\hline$\stackrel{2}{\frac{2}{二}}$ & Otu1275 & Picobiliphyta sp. & 94 & & 4.8 & 5.4 & & 8.5 & 4.1 & 10.0 & & 4.7 & 3.2 \\
\hline$\frac{0}{0}$ & Otu1283 & Picobiliphyta sp. & 100 & & 4.8 & 2.7 & & 2.6 & 6.5 & & & 3.4 & 4.3 \\
\hline$\frac{5}{2}$ & Otu1792 & Picobiliphyta sp. & 100 & & & & 27.3 & 0.9 & 4.1 & 7.5 & 16.7 & 3.9 & \\
\hline & Otu1780 & Telonemia-Group-1 sp. & 97 & 57.8 & 66.7 & 7.1 & & 25.0 & 16.7 & & & 93.3 & 42.9 \\
\hline & Otu0011 & Telonemia-Group-2 sp. & 100 & 16.5 & & & & 50.0 & 30.0 & 75.0 & & & 57.1 \\
\hline & Otu1445 & Telonemia-Group- $2 \mathrm{sp}$. & 97 & 13.8 & 13.3 & 28.6 & & 25.0 & 13.3 & & & 6.7 & \\
\hline & Otu1575 & Telonemia-Group-2 sp. & 98 & & & & & & & 25.0 & 50.0 & & \\
\hline হo & Otu0345 & Telonemia-Group-2 sp. & 100 & 8.3 & 13.3 & 42.9 & & & 10.0 & & & & \\
\hline$\stackrel{n}{0}$ & Otu0040 & Telonemia-Group-2 sp. & 97 & & & 7.1 & 50.0 & & & & & & \\
\hline$\widetilde{\sigma}$ & Otu0585 & Telonemia-Group-1 sp. & 99 & 3.7 & 6.7 & 7.1 & & & 16.7 & & & & \\
\hline $\bar{\varepsilon}$ & Otu0140 & Telonemia-Group-2 sp. & 97 & & & 7.1 & & & 6.7 & & & & \\
\hline 듬 & Otu0075 & Telonemia-Group-2 sp. & 100 & & & & & & & & & & \\
\hline $\bar{\omega}$ & Otu0732 & Telonemia-Group-2 sp. & 97 & & & & 50.0 & & 6.7 & & & & \\
\hline & Otu1863 & Exobasidiomycetes $s p$. & 100 & & 83.3 & & 100.0 & 66.7 & & 6.7 & 15.4 & 1.0 & 29.6 \\
\hline & Otu0879 & Candida austromarina & 100 & & & & & & & & & & 17.9 \\
\hline & Otu1430 & Laccocephalum mylittae & 88 & & & & & 12.5 & & & & & 8.2 \\
\hline & Otu0716 & E obasidiomycetes sp. & 100 & & & & & 12.5 & & & & & 13.6 \\
\hline & Otu1424 & Coccodinium bartschii & 95 & & & & & & 14.3 & & 7.7 & & 17.2 \\
\hline & Otu1290 & Lecythophora mutabilis & 100 & & & & & 2.8 & 28.6 & 25.0 & 53.8 & & \\
\hline & Otu0127 & Acremonium antarcticum & 99 & & & & & & & & & & \\
\hline & Otu0945 & Phaeosphaeria nodorum & 97 & & & & & & & & & & \\
\hline & Otu0889 & Saccharomyces sp. & 100 & & & & & & & & & & 5.5 \\
\hline $\bar{\alpha}$ & Otu0167 & Glaciozyma antarctica & 98 & & & & & & & & & & \\
\hline ¿े & Otu0475 & Cryptococcus gastricus & 99 & & & & & & & & & & 5.2 \\
\hline 'ָס & Otu1631 & Rhodosporidium sphaerocarpum & 100 & & & & & & & 1.7 & & & 1.4 \\
\hline \begin{tabular}{|c|c|} 
\\
\end{tabular} & Otu1433 & Pyrenophora tritici-repentis & 100 & & & & & 5.6 & & & & & \\
\hline
\end{tabular}


Table 4. Continued.

\begin{tabular}{|c|c|c|c|c|c|c|c|c|c|c|c|c|c|c|c|c|c|c|c|}
\hline \multirow{8}{*}{ 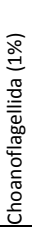 } & Otu1941 & StephanoecidaeGroupD sp. & 97 & 22.2 & 10.0 & 11.1 & 25.0 & 33.3 & 44.1 & 25.0 & & 83.3 & 4.0 & 21.5 & & 73.3 & 66.7 & 4.0 & 25.0 \\
\hline & Otu1928 & Stephanoeca cauliculata & 99 & 57.5 & 72.7 & 55.6 & 25.0 & & 11.8 & 25.0 & & 16.7 & 1.0 & 1.8 & & 13.3 & 16.7 & 6.0 & 25.0 \\
\hline & Otu1710 & StephanoecidaeGroupD sp. & 100 & 18.5 & 18.2 & & & & 23.5 & & 66.7 & & 5.0 & 43.8 & & 6.7 & 13.3 & & 25.0 \\
\hline & Otu0960 & StephanoecidaeGroupH sp. & 90 & & & & & 66.7 & 2.9 & 25.0 & & & & 24.6 & & & & & \\
\hline & Otu1959 & StephanoecidaeGroupD sp. & 95 & & & 11.1 & & & 11.8 & & & & & & & & 3.3 & & 25.0 \\
\hline & Otu1706 & StephanoecidaeGroupD sp. & 93 & 1.9 & & & & & & 25.0 & 33.3 & & & & & 6.7 & & & \\
\hline & Otu1905 & StephanoecidaeGroupH sp. & 91 & & & 22.2 & & & & & & & & & & & & & \\
\hline & Otu1828 & StephanoecidaeGroupD sp. & 94 & & & & 5.0 & & & & & & & & & & & & \\
\hline \multirow{10}{*}{ 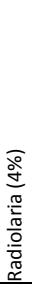 } & Otu1699 & Spumellarida-Group-I sp. & 99 & & & & 67.9 & & 4.2 & 8.0 & 73.7 & & & & 18.8 & & & & 50.7 \\
\hline & Otu1655 & Spumellarida-Group-I sp. & 100 & & & & & 16.7 & 1.1 & 4.0 & 13.9 & & & & & & & & 1.0 \\
\hline & Otu1138 & Stylodictya sp. & 99 & & & & 1.9 & 25.0 & 42.1 & 16.0 & 0.4 & & & & 37.5 & 33.3 & & & 7.7 \\
\hline & Otu1589 & Spumellarida-Group-I sp. & 100 & & & & & & & 4.0 & 6.7 & & & & & & & & 0.3 \\
\hline & Otu0699 & Triastrum aurivillii & 95 & & 25.0 & & & & 1.1 & & & & & 76.2 & & & & & 1.7 \\
\hline & Otu1856 & RAD-B-Group-IV sp. & 99 & & & 50.0 & 1.9 & & 12.6 & 8.0 & 0.3 & & & & 6.3 & 33.3 & & 66.7 & 5.8 \\
\hline & Otu0036 & RAD-B-Group-IV sp. & 97 & & & & 1.9 & & & 8.0 & & & & & & & & & 7.5 \\
\hline & Otu0686 & RAD-B-Group-II sp. & 100 & & & & & & & & & & & & & & & & 7.4 \\
\hline & Otu1654 & RAD-B-Group-II sp. & 99 & & & & 5.7 & & 2.1 & 4.0 & & & & & & & & & 5.8 \\
\hline & Otu1349 & RAD-B-Group-IV sp. & 100 & & 75.0 & & & & 17.9 & 16.0 & & & & 19.0 & & & 100.0 & 33.3 & \\
\hline \multirow{15}{*}{ 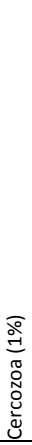 } & Otu1449 & Protocystis iphodon & 100 & & & & & 2.6 & & & & & & 100.0 & 100.0 & & & & \\
\hline & Otu1378 & Protaspa-lineage sp. & 98 & & & 100.0 & & 30.8 & 26.7 & & & 6.7 & & & & 33.3 & 28.6 & 33.3 & 100.0 \\
\hline & Otu1257 & Ebria tripartita & 100 & & & & & 51.3 & 20.0 & & & & & & & & & & \\
\hline & Otu0591 & Protaspa-lineage sp. & 99 & & & & & 2.6 & 6.7 & & & 46.7 & 13.3 & & & & 28.6 & & \\
\hline & Otu0887 & TAGIRI1-lineage sp. & 98 & & & & & & & & & & 40.0 & & & & 28.6 & & \\
\hline & Otu1806 & Protaspa sp. & 99 & & & & 100.0 & 10.3 & & & & & & & & 16.7 & & & \\
\hline & Otu0881 & Cryothecomonas-lineage sp. & 99 & & & & & & & & & & 33.3 & & & & & & \\
\hline & Otu0170 & Cryothecomonas-lineage $s p$. & 100 & & & & & & 6.7 & & & & & & & 33.3 & & 33.3 & \\
\hline & Otu0742 & TAGIRI1-lineage sp. & 98 & & & & & & & & & & 13.3 & & & 16.7 & 14.3 & & \\
\hline & Otu0201 & Cryothecomonas sp. & 100 & & & & & & 20.0 & & & & & & & & & & \\
\hline & Otu1040 & Mataza-lineage sp. & 100 & & & & & 2.6 & & & & 13.3 & & & & & & & \\
\hline & Otu1368 & Protaspa-lineage sp. & 100 & & & & & & 6.7 & & & 6.7 & & & & & & 33.3 & \\
\hline & Otu0857 & Cryothecomonas sp. & 99 & & & & & & & & & 13.3 & & & & & & & \\
\hline & Otu0941 & Marimonadida sp. & 92 & & & & & & & & & 13.3 & & & & & & & \\
\hline & Otu1624 & Endo4-lineage sp. & 99 & & & & & & & & 100.0 & & & & & & & & \\
\hline \multirow{10}{*}{ 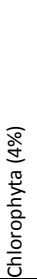 } & Otu1717 & Micromonas pusilla (RCC658) & 100 & 2.0 & 28.8 & 3.5 & & 25.4 & 38.6 & 68.4 & & 1.0 & 6.6 & 5.2 & & & 5.9 & 3.3 & \\
\hline & Otu1962 & Pyramimonas gelidicola & 97 & 6.9 & 4.0 & 2.3 & & 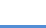 & 0.9 & & & 1.8 & 72.5 & 29.6 & & 17.4 & 2.9 & 53.3 & \\
\hline & Otu1742 & Micromonas pusilla (RCC418) & 100 & 22.7 & 29.9 & 3.5 & & 13.6 & 21.6 & 5.3 & & 30.0 & 6.1 & & & & & 3.3 & \\
\hline & Otu1918 & Bathycoccus prasinos & 100 & 12.8 & 26.6 & 34.5 & & 6.8 & 16.5 & & & 10.0 & 4.4 & 1.0 & & & 17.6 & & \\
\hline & Otu1791 & Prasinoderma coloniale & 95 & 33.5 & 3.4 & & 5.0 & 8.5 & 2.5 & 15.8 & & & 0.9 & 3.8 & & & & 3.3 & \\
\hline & Otu0166 & Pyramimonas disomata & 96 & 2.7 & 1.1 & & 5.0 & & & & & & & 33.3 & & & & 6.7 & \\
\hline & Otu1766 & Pyramimonas olivacea & 99 & 2.2 & 1.7 & 1.4 & & 3.4 & 2.2 & & 1.0 & 5.5 & & 18.5 & & 47.8 & 14.8 & 6.7 & 48.3 \\
\hline & Otu0940 & Pyramimonas sp. & 100 & & 0.6 & 0.7 & & 1.2 & 1.5 & & & 13.6 & 4.4 & 4.6 & & 13.4 & 41.2 & 1.0 & \\
\hline & Otu1775 & Crustomastigaceae sp. & 99 & 0.2 & & & & 16.9 & 0.4 & 5.3 & & 10.0 & 1.3 & 3.6 & & & 17.6 & & 51.7 \\
\hline & Otu0151 & Mamiella sp. & 100 & & & 0.7 & & 6.7 & 2.5 & & & 6.3 & 1.7 & & & 4.3 & & & \\
\hline
\end{tabular}

Potential limitation regarding pyrosequencing detection of Bacillariophyceae have been reported recently in an extensive study at the San Pedro Ocean Time Series station (SPOT, Lie et al., 2013). They can be related to extraction efficiency from thick walled diatoms (Medinger et al., 2010) and/or amplification biases favouring species with high 18S rRNA gene copy number, such as ciliates and dinoflagellates (Potvin and Lovejoy, 2009). It is also worth noting that 28 out of the 52 taxa identified by microscopy (Armand et al., 2008) were not referenced in the GenBank. Finally, regarding the 27 diatom taxa that were "identified" only by pyrosequencing - based on sequence similarity with the closest existing cultured relatives in GenBank - they mainly belonged to the genera previously observed in this area (Armand et al., 2008). The accuracy of BLAST-derived taxonomy, especially at low-level taxa, depends on sequence length, variability of the $18 \mathrm{~S}$ region, database coverage for the specific taxonomic group, and correct identification of the reference sequence (Bik et al., 2012).

Sequences belonging to the nano- and picophytoplanktonic groups of Bolidophyceae, Pelagophyceae,
Chrysophyceae and Cryptophyta were found at relatively low abundances in all samples. Moreover, Haptophyta were dominated by an OTU affiliated as Phaeocystis antarctica (100\% sequence identity). This phylotype has been previously reported as dominant in the south of the polar front (Wolf et al., 2014), in the Ross Sea waters (DiTullio et al., 2000), and in the naturally iron-fertilized bloom around the Crozet Plateau (Poulton et al., 2007).

\subsubsection{Microzooplankton: Dinoflagellates, ciliates and radiolaria}

Although Dinophyceae might be over-represented in the sequence data, possibly due to its high $18 \mathrm{~S}$ gene copy number (e.g. Prokopowich et al., 2003; Zhu et al., 2005), tag pyrosequencing has made possible the highlighting of its extensive diversity (161 OTUs) in the Southern Ocean - previously missed by conventional microscopy and/or pigment analysis (see also Wolf et al., 2014). For example, based on microscopy, Gyrodinium is the most abundant dinoflagellate analysed; however, no reliable distinction has been made 
Table 5. Results of SIMPER (similarity percentages) following the Bray-Curtis cluster analysis (Fig. 6a). Forty-one OTUs contributing for at least $1 \%$ of the similarity of each cluster are listed in this table. In parentheses, the mean of Bray-Curtis similarity is given for each cluster.

\begin{tabular}{|c|c|c|c|c|c|c|}
\hline OTUs & $\begin{array}{l}\text { Taxonomic } \\
\text { groups }\end{array}$ & $\begin{array}{l}\text { Putative taxonomic } \\
\text { affiliation }\end{array}$ & $\begin{array}{l}\text { Cluster (i) } \\
(43.8 \%)\end{array}$ & $\begin{array}{l}\text { Cluster (ii) } \\
(51.8 \%)\end{array}$ & $\begin{array}{l}\text { Cluster (iii) } \\
(47.6 \%)\end{array}$ & $\begin{array}{l}\text { Cluster (iv) } \\
(20.7 \%)\end{array}$ \\
\hline Otu1951 & Dinophyceae & Gyrodinium spirale & 5.1 & 17 & 33.5 & 1.3 \\
\hline Otu1914 & & Gyrodinium rubrum & & 5.2 & 17.9 & \\
\hline Otu1967 & & Gymnodinium sp. & 8.8 & 3.5 & 2.6 & 4.9 \\
\hline Otu1898 & & Karlodinium micrum & & 5.9 & 1.9 & 1.3 \\
\hline Otu1770 & & Warnowia sp. & & 3 & 1.5 & 4.1 \\
\hline Otu1016 & & Gyrodinium rubrum & 1.3 & 3.3 & & 3.6 \\
\hline Otu1763 & & Dinophyceae sp. & 1.5 & 3.7 & & 1.5 \\
\hline Otu1808 & & Warnowia sp. & 1.8 & 3.6 & & \\
\hline Otu1953 & & Peridinium tyrrhenicum & & 1.4 & & 1.8 \\
\hline Otu1871 & & Gymnodinium catenatum & & & & 1.7 \\
\hline Otu1816 & & Karlodinium micrum & & & & 1.3 \\
\hline Otu1722 & & Gymnodinium sp. & & 1.3 & & \\
\hline Otu1454 & & Islandinium minutum & & 1.3 & & \\
\hline Otu1793 & & Amphidinium semilunatum & & & 1.1 & \\
\hline Total & & & 18.4 & 49.2 & 58.6 & 21.5 \\
\hline Otu1653 & MALV-I & Dino-Group-I-Clade-1 sp. & 1.3 & 2.2 & 2.1 & 6 \\
\hline Otu1912 & & Dino-Group-I-Clade-1 sp. & & 1.9 & 3.2 & 1.4 \\
\hline Otu1292 & & Dino-Group-I-Clade-5 sp. & & 2.9 & & 1.6 \\
\hline Otu1285 & & Dino-Group-I-Clade-1 sp. & 1.3 & & 2.6 & \\
\hline Otu1790 & & Dino-Group-I-Clade-4 sp. & 1.2 & & 1.4 & \\
\hline Otu1393 & & Dino-Group-I-Clade-1 sp. & 1 & & & \\
\hline Total & & & 4.7 & 6.9 & 9.2 & 9 \\
\hline Otu1211 & MALV-2 & Dino-Group-II-Clade-10 sp. & & & & 3.7 \\
\hline Otu1116 & & Dino-Group-II-Clade-10 sp. & & & & 3.2 \\
\hline Otu1663 & & Dino-Group-II-Clade-7 sp. & & & & 2 \\
\hline Otu1613 & & Dino-Group-II-Clade-7 sp. & & & & 2 \\
\hline Otu1513 & & Dino-Group-II-Clade-6 sp. & & & & 1.1 \\
\hline Otu1183 & & Dino-Group-II-Clade-7 sp. & & & & 1.1 \\
\hline Total & & & & & & 13.1 \\
\hline Otu1799 & Ciliophora & Strombidium biarmatum & 1.2 & & & \\
\hline Otu1447 & Bacillariophyceae & Thalassiosira tenera & & 6.4 & 2 & 1.9 \\
\hline Otu0978 & & Thalassiosira delicatula & & 2.7 & & \\
\hline Total & & & & 9.1 & 2 & 1.9 \\
\hline Otu1932 & Pelagophyceae & Aureococcus anophagefferens & 4.7 & & & \\
\hline Otu1762 & MAST & MAST-1B sp. & 1.3 & & & \\
\hline Otu1923 & & MAST-1C sp. & 1.1 & & & \\
\hline Total & & & 2.4 & & & \\
\hline Otu1717 & Chlorophyta & Micromonas pusilla & 4 & & & \\
\hline Otu1918 & & Bathycoccus prasinos & 3.8 & & & \\
\hline Otu1742 & & Micromonas pusilla & 3.8 & & & \\
\hline Total & & & 11.5 & & & \\
\hline Otu1782 & Haptophyta & Phaeocystis antarctica & 18.3 & 2.7 & 5.2 & 1.3 \\
\hline Otu1884 & & Chrysochromulina strobilus & 4.5 & & & \\
\hline Otu1907 & & Chrysochromulina sp. & 2.7 & & 1.3 & \\
\hline Total & & & 25.5 & 2.7 & 6.4 & 1.3 \\
\hline Otu1863 & Fungi & Malassezia restricta & 1.3 & & & \\
\hline Otu1699 & Radiolaria & Spumellarida sp. & & & & 6.2 \\
\hline Otu1138 & & Stylodictya sp. & & & & 1 \\
\hline Total & & & & & & 7.2 \\
\hline
\end{tabular}




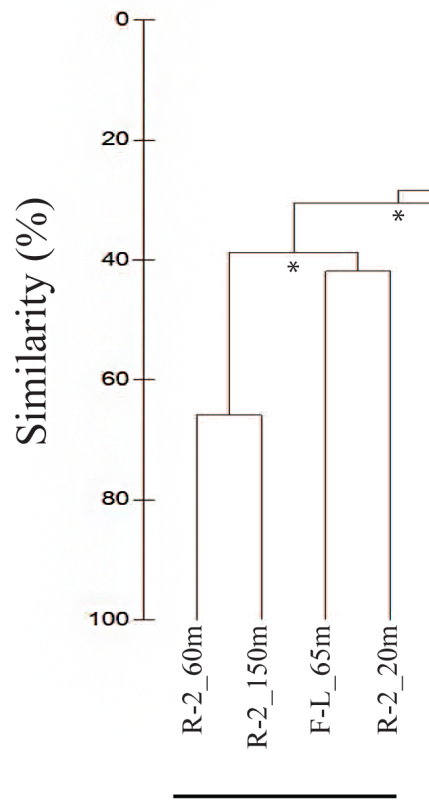

(i)

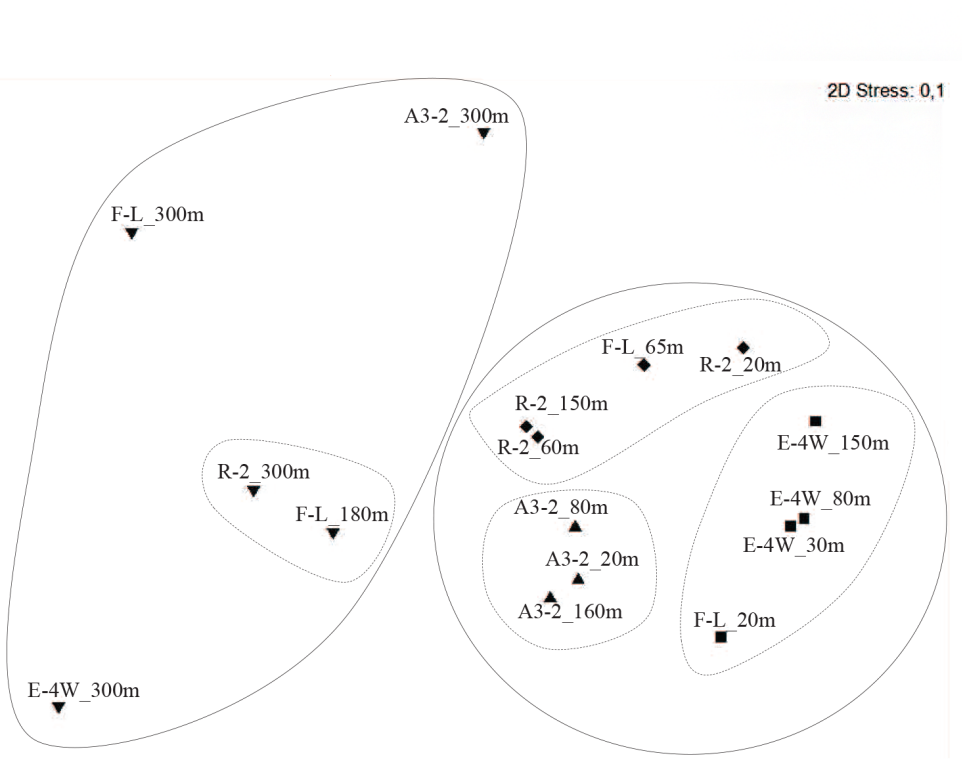

(a)

(b)

Figure 6. Cluster diagram for the 16 samples constructed from a Bray-Curtis similarity matrix of square-root-transformed OTU abundances. Asterisks at nodes in the dendrogram indicate significant differences between bifurcations $(P<0.05)$ (a). Nonmetric multidimensional (nMDS) scaling plots in two dimensions constructed from a Bray-Curtis similarity matrix. Bray-Curtis similarity contours are $15 \%$ (solid lines) and $40 \%$ (dashed lines) (b).

between G. spirale and G. rubrum with morphological observations (Saito et al., 2005; Georges et al., unpublished KEOPS2 data)

Ciliophora, which are ecologically important grazers of small-sized phytoplankton, accounted for a relative high number of OTUs (60 OTUs). As with previous microscopic observations in the Kerguelen area (Christaki et al., 2008), the most representative ciliate sequences in this study belonged to Strombidiidae. The relatively large-sized Strom- bidium spp. ( $\geq 50 \mu \mathrm{m})$ can be plastidic (mixotrophic) and along with Tontonia spp. and Laboea spp. - also present in sequences - were found to contribute to $40-60 \%$ of the aloricate ciliate biomass during the late bloom on the Kerguelen plateau (KEOPS1, Christaki et al., 2008). Finally, the most relatively abundant sequences of tintinnid taxa - which are also important nanophytoplankton consumers - belonged to the large Cymatocyclis calyciformis (Christaki et al., 2008). 
Radiolaria were another well-represented microzooplankton group (35 OTUs). These can act as particle feeders, by trapping their prey on the peripheral network of rhizopodia, or capture diatoms. They are also hosts of dinoflagellate symbionts and parasites, and may be important reservoirs of MALV taxa (e.g. Bråte et al., 2012). In this study, the relative increase of MALV with depth was consistent with a parallel increase of Radiolaria. This observation is also supported by the hypothesis that MALV taxa are able to parasitize "deeper" planktonic organisms such as Spumellarida (Guillou et al., 2008), which were the most common group and were always well represented in the deeper water samples in this study (Fig. 4, Table 4). Radiolaria and MALV taxa characterizing deeper protistan assemblages have also been reported in the North Atlantic (Countway et al., 2007; 2010; Not et al., 2007) and deep Antarctic polar front samples (López-Garcia et al., 2001).

\subsubsection{Symbionts, parasites, and decomposers}

This assemblage included the taxonomic groups of MALVI, MALV-II, Labyrinthulomycetes, Pirsonia, Oomyeta, Apicomplexa, Perkinsea, Fungi and Cercozoa. Many of these groups have a zooflagellate stage in their life cycles, and are classified together in microscopical studies as "heterotrophic nanoflagellates". MALV-I and MALV-II appear in virtually all marine surveys (López-Garcia et al., 2001; Massana and Pedrós-Alió, 2008). Their considerable abundance and diversity suggests interactions with various hosts, and therefore it has been proposed that the whole MALV assemblage is composed of marine parasites (Skovgaard et al., 2005; Massana and Pedrós-Alió, 2008).

Fungi and Cercozoa accounted for 28 and 17 OTUs, respectively. In a recent succession study in the English Channel, it was observed that these groups mostly co-occurred with Bacillariophyceae (Christaki et al., 2014). Fungi are possibly related to the polysaccharide degradation of the freshly produced organic material by primary producers (Kimura and Naganuma 2001; Raghukumar, 2004). It is known for diatoms that polysaccharides are their main exudates (Myklestad, 1995 and references therein), and these sugars could promote the growth of Fungi. Many Cercozoa are parasites of marine organisms, including large heavily silicified diatoms (e.g. Tillman et al., 1999; Schnepf and Kühn, 2000), which could explain why Fungi and Cercozoa were detected in the bloom stations and were poorly represented (2-3 OTUs, Table 4) at the HNLC R-2 station. Labyrinthulomycetes were also better represented in terms of numbers of sequences in the bloom stations (Table 4). Labyrinthulomycetes (19 OTUs) are common osmoheterotrophic marine protists (López-Garcia et al., 2001) having parasitic, commensalistic, or mutualistic relationships with their hosts.
They play an important role in decomposition processes (Collado-Mercado et al., 2010) by colonizing fecal pellets, including under deep-sea conditions (Raghukumar, 2004).

\subsubsection{Small heterotrophic protists}

Among the small heterotrophic protists found in the samples, there were a variety of MAST (46 OTUs), Choanoflagellida (10 OTUs) and Telonemia (12 OTUs). MAST taxa are widely distributed in the world's oceans, and have been identified as free-living bacterivorous heterotrophic flagellates through a combination of FISH and other measurements (Massana et al., 2006; Jürgens and Massana, 2008 for a review). Choanoflagellida of the genus Stephanotheca sp. were also observed by epifluorescence microscopy in KEOPS2 samples, and were more abundant and diversified in the 0 $200 \mathrm{~m}$ layer (Georges et al., unpublished KEOPS2 data).

\subsection{Variability of protistan assemblages relative to iron fertilization}

In general, the stability of OTUs richness and diversity indices between the HNLC R-2 and iron-fertilized stations indicated that the environment maintained an overall diversity across stations and depths (Table 2). These observations are in agreement with previous molecular studies based on protistan diversity (e.g. Countway et al., 2007; Monchy et al., 2012). However, community structure analysis showed clear differences inside and outside the blooms (Fig. 6a).

\subsubsection{HNLC station}

Based on trophic organization, HNLC areas seem conceptually similar to oligotrophic regions dominated by small producers and an active microbial food web (e.g. Hall and Safi, 2001; Oliver et al., 2004; Christaki et al., 2014, 2008; Obernosterer et al., 2008). The characteristic contributors of the HNLC cluster (i) were Haptophyta, Chlorophyta and MAST, which included mainly nanoplanktonic organisms. During KEOPS2, the relative importance of small-sized cells at the HNLC station is in accordance with the flow cytometry data $\left(4.8 \pm 1.910^{3} \mathrm{~mL}^{-1}\right.$ nano-picophytoplankton cells in comparison to $1.8 \pm 1.310^{3} \mathrm{~mL}^{-1}$ at the bloom stations; KEOPS2 data). The factors influencing phytoplankton community composition (e.g. diatoms vs. Phaeocystis sp.) in the Southern Ocean are a complex interplay between bottomup (iron-silicate-light availability; controlling growth) and top-down effects (grazing; controlling mortality) (Cullen, 1991; Arrigo et al., 1999; Smetacek et al., 2004; Schoemann et al., 2005). Live plankton observations completed on board (https://www.youtube.com/watch?v=KPgoz8bWRJU) revealed the presence of small colonies and free-living cells belonging to the Haptophyta Phaeocystis sp. at all stations. It seems that Phaeocystis species cope best with the environmental conditions in the open ocean waters south of the Polar 
Front, where it was found to be the most dominant phylotype (Wolf et al., 2014).

\subsubsection{Iron-fertilized sites}

The mechanisms that fertilize the surface water in the region around Kerguelen are complex, which results in a patchwork of blooms with diverse biological and biogeochemical response. The phytoplankton bloom at the "historical" A3 station situated on the Kerguelen Plateau is bottom-up sustained by low-level supplies of iron and other nutrients (Blain et al., 2007). Drifters have revealed a northeastward-driven circulation pattern in the Kerguelen Plateau and oceanic area, while strong horizontal mixing have been found in the East Kerguelen Basin off the plateau (Zhou et al., 2014; Fig. 1b). Station E-4W is located at the shelf break in a region with very strong currents (Zhou et al., 2014), and consequently receives ironrich waters from the Kerguelen Island and Plateau (A3 station area) which mix with Polar Front waters that cross the Kerguelen Plateau while traveling northeast (Fig. 1b). The depth of the ML varied considerably, from $40 \mathrm{~m}$ north of the Polar Front at station F-L to $170 \mathrm{~m}$ above the plateau at station A3. In accordance with these hydrographic characteristics, multivariate analysis of sequences showed that the ML sample of the F-L $(20 \mathrm{~m})$ was found in the same cluster as the E-4W samples, while the $65 \mathrm{~m}$ F-L sample was grouped with the HNLC samples. The OTUs putatively affiliated to heterotrophic dinoflagellate taxa (Table 5) were the major contributors of clusters (ii) and (iii) (Fig. 6a, b). Dinoflagellate increase during iron-fertilized blooms - in particular, Gyrodinium spp. has been observed with microscopic counts during the iron addition experiments, and has been attributed to the increase of their diatoms prey (Hall and Safi, 2001; Saito et al., 2005; Henjes et al., 2007).

Concluding, the tag pyrosequening approach in this study has provided an overview of the protistan assemblages present in the naturally fertilized blooms and the HNLC waters in the Southern Ocean. Despite the under-representation of Bacillariophyceae diversity and the over-representation of Dinophyceae in the sequences, the community similarity analysis showed clear differences between the iron-fertilized and the HNLC waters, and among the blooms, in regard to their location and the fertilization mechanisms. The molecular approach has also highlighted a rich assemblage of potential phytoplankton parasites and organic matter decomposers mostly present in the iron-fertilized blooms.

The Supplement related to this article is available online at doi:10.5194/bg-11-5847-2014-supplement.
Acknowledgements. KEOPS was financed by INSU-CNRS, IPEV and ANR and the French Ministry of Higher Education through a PhD grant to C. Georges. We thank our many colleagues who participated in the collection of various data sets, the KEOPS co-ordinator S. Blain, the chief scientist on board B. Quéguiner and the crew aboard the R/V Marion Dufresne for their help in the successful completion of the cruise. We also thank www.englisheditor.webs.com for the paper's English proofing.

Edited by: G. Herndl

\section{References}

Altschul, S. F., Gish, W., Miller, W., Myers, E. W., and Lipman D. J.: Basic local alignment search tool, J. Mol. Biol., 215, 403-410, 1990.

Amann, R. I., Binder, B. J., Olson, R. J., Chisholm, S. W., Devereux, R., and Stahl, D. A.: Combination of 16S rRNA-targeted oligonucleotide probes with flow cytometry for analyzing mixed microbial populations, Appl. Environ. Microbiol., 56, 1919-192, 1990.

Armand, L. K., Crosta, X., Quéguiner, B., Mosseri, J., and Garcia, N.: Diatoms preserved in surface sediments of the northeastern Kerguelen Plateau, Deep Sea Res. II, 55, 677-692, 2008.

Arrigo, K. R., Robinson, D. H., Worthen, D. L., Dunbar, R. B., DiTullio, G. R., VanWoert, M., and Lizotte, M. P.: Phytoplankton community structure and the drawdown of nutrients and $\mathrm{CO}_{2}$ in the Southern Ocean, Science, 283, 365-367, 1999.

Behnke, A., Engel, M., Christen, R., Nebel, M., Klein, R. R., and Stoeck, T.: Depicting more accurate pictures of protistan community complexity using pyrosequencing of hypervariable SSU rRNA gene regions, Environ. Microbiol., 13, 340-349, 2010.

Bik, H. M., Porazinska, D. L., Creer, S., Caporaso, J. G., Knight, R., and Thomas, K. W.: Sequencing our ways towards understanding global eukaryotic biodiversity, Trends Ecol. Evol., 27, 233-243, 2012.

Blain, S., Quéguiner, B., Armand, L., Belviso, S., Bombled, B., Bopp, L., Bowie, A., Brunet, C., Brussaard, C., Carlotti, F., Christaki, U., Corbiere, A., Durand, I., Ebersbach, F., Fuda, J. L., Garcia, N., Gerringa, L., Griffiths, B., Guigue, C., Guillerm, C., Jacquet, S., Jeandel, C., Laan, P., Lefevre, D., Lo Monaco, C., Malits, A., Mosseri, J., Obernosterer, I., Park, Y. H., Picheral, M., Pondaven, P., Remenyi, T., Sandroni, V., Sarthou, G., Savoye, N., Scouarnec, L., Souhaut, M., Thuiller, D., Timmermans, K., Trull, T., Uitz, J., van Beek, P., Veldhuis, M., Vincent, D., Viollier, E., Vong, L., and Wagener, T.: Effect of natural iron fertilization on carbon sequestration in the southern ocean, Nature, 446, 10701074, 2007.

Blain, S., Quéguiner, B., and Trull, T.,: The natural iron fertilization experiment KEOPS (Kerguelen Ocean and Plateau compared Study): an overview, Deep-Sea Res. II, 55, 559-565, 2008.

Blain, S., Oriol, L., Capparos, J., Guéneuguès, A., and Obernosterer, I.: Distributions and stoichiometry of dissolved nitrogen and phosphorus in the iron fertilized region near Kerguelen (Southern Ocean), this volume.

Boyd, P. W., Law, C. S., Wong, C. S., Nojiri, Y., Tsuda, A., Levasseur, M.,Takeda, S., Rivkin, R., Harrison, P.J., Strzepek, R., Gower, J.,McKay, R. M., Abraham, E., Arychuk, M., BarwellClarke, J.,Crawford, W., Crawford, D., Hale, M., Harada, K., 
Johnson, K., Klyosawa, H., Kudo, I., Marchetti, A., Miller, W., Needoba, J., Nishioka, J., Ogawa, H., Page, J., Robert, M., Saito, H., Sastri, A., Nelson, S., Soutar, T., Sutherland, N., Taira, Y., Whitney, F., Wong, S.-K. E., and Yoshimura, T.: The decline and fate of an iron-induced subarctic phytoplankton bloom, Nature, 428, 549-553, 2004.

Boyd, P. W., Jickells, T., Law, C. S., Blain, S., Boyle, E. A., Buesseler, K. O., Coale, K. H., Cullen, J. J., de Baar, H. J. W., Follows, M., Harvey, M., Lancelot, C., Levasseur, M., Owens, N. P. J., Pollard, R., Rivkin, R. B., Sarmiento, J., Schoemann, V., Smetacek, V., Takeda, S., Tsuda, A., Turner, S., and Watson, A. J.: Mesoscale iron enrichment experiments 1993-2005: Synthesis and future directions, Science, 315, 612-617, 2007.

Bråte, J., Krabberød, A. K., Dolven, J. K., Ose, R. F., Kristensen, T., Bjørklund, K. R.: Radiolaria associated with large diversity of marine alveolates, Protist, 163, 767-777, 2012.

Brussaard, C. P. D., Timmermans, K. R., Uitz, J., and Veldhuis, M. J. W.: Virioplankton dynamics and virally induced phytoplankton lysis versus microzooplankton grazing southeast of the Kerguelen (Southern Ocean), Deep-Sea Res. II, 55, 752-765, 2008.

Caron, D. A., Countway, P. D., Savai, P., Gast, R. J., Schnetzer, A., Moorthi, S. D, Dennett M. R., Moran, D. M. and Jones, A. C.: Defining DNA-based operational taxonomic units for microbialeukaryote ecology, Appl. Environ. Microbiol., 75, 5797-5808, 2009

Caron, D. A., Countway, P. D., Jones, A. C., Kim, D. Y., and Schnetzer, A.: Marine protistan diversity, Ann. Rev. Mar. Sci., 4, 467493, 2012.

Christaki, U., Obernosterer, I., Van Wambeke, F., Veldhuis, M. J. W., Garcia, N., and Catala, P.: Microbial food web structure in a naturally iron fertilized area in the Southern Ocean (Kerguelen Plateau), Deep-Sea Res. II, 55, 706-719, 2008.

Christaki, U., Kormas, K. A., Genitsaris, S., Georges, C., SimeNgando, T., Viscogliosi, E., and Monchy, S.: Winter-summer succession of unicellular eukaryotes in a meso-eutrophic coastal system, Microb. Ecol., 67, 13-23, this volume.

Christaki, U., Lefèvre, D., Georges, C., Colombet, J., Catala, P., Courties, C., Sime-Ngando, T., Blain, S., and Obernosterer I.: Microbial food web dynamics during spring phytoplankton blooms in the naturally iron-fertilized Kerguelen area (Southern Ocean), 2014, this volume.

Clarke, K. R.: Non-parametric multivariate analyses of changes in community structure, Austr. J. Ecol., 18, 117-143, 1993.

Closset, I., Lasbleiz, M., Leblanc, K., Quéguiner, B., Cavagna, A.J., Elskens, M., Navez, J., and Cardinal D.: Seasonal evolution of net and regenerated silica production around a natural $\mathrm{Fe}$ fertilized area in the Southern Ocean estimated from Si isotopic approaches, this volume.

Collado-Mercado E., Radway, J. C., and Collier, J. L.: Novel uncultivated labyrinthulomycetes revealed by $18 \mathrm{~S}$ rDNA sequences from seawater and sediment samples, Aquat. Microb. Ecol., 58, 215-228, 2010.

Countway, P. D., Gast, R. J., Dennett, M. R., Savai, P., Rose J. M., and Caron, D. A.: Distinct protistan assemblages characterize the euphotic zone and deep sea $(2500 \mathrm{~m})$ of the western North Atlantic (Sargasso Sea and Gulf Stream), Environ. Microbiol., 9, 1219-1232, 2007.

Countway, P. D., Vigil, P. D., Schnetzer, A., Moorthi, S. D., and Caron, D. A.: Seasonal analysis of protistan community struc- ture and diversity at the USC Microbial Observatory (San Pedro Channel, North Pacific Ocean), Limnol. Oceanogr., 55, 23812396, 2010.

Cullen, J. J.: Hypotheses to explain high-nutrient conditions in the open sea, Limnol. Oceanogr., 36, 1578-1599, 1991.

DiTullio, G. R., Grebmeier, J. M., Arrigo, K. R., Lizotte, M. P., Robinson, D. H., Leventer, A., Barry, J. P., VanWoert, M. L., and Dunbar, R. B.: Rapid and early export of Phaeocystis Antarctica blooms in the Ross Sea, Antarctica, Nature, 404, 595-598, 2000.

Edgar, R. C.: Search and clustering orders of magnitude faster than BLAST, Bioinformatics, 26, 2460-2461, 2010.

Edgcomb, V., Orsi, W., Bunge, J., Jeon, S., Christen, R., Leslin, C., Holder, M., Taylor, G. T., Suarez, P., Varela, R., and Epstein, S.: Protistan microbial observatory in the Cariago Basin, Caribbean, I. Pyrosequencing vs. Sanger insights into species richness, ISME J., 5, 1344-1356, 2011.

Gilbert, J. A., Steele, J. A., Caporaso, J. G., Steinbruck, L., Reeder, J., Temperton, B., Huse, S., McHardy, A. C., Knight, R., Joint, I., Somerfield, P., Fuhrman, J. A., and Field, D.: Defining seasonal marine microbial community dynamics, ISME J., 6, 298-308, 2012.

Guillou, L., Viprey, M., Chambouvet, A., Welsh, R. M., Kirkham, A. R., Massana, R., Scanlan, D. J., and Worden A. Z.: Widespread occurrence and genetic diversity of marine parasitoids belonging to Syndiniales (Alveolata), Environ. Microbiol., 10, 3349-3365, 2008.

Guillou, L., Bachar, D., Audic, S., Bass, D., Berney, C., Bittner, L., Boutte, C., Burgaud, G., de Vargas, C., Decelle, J., Del Campo, J., Dolan, J. R., Dunthorn, M., Edvardsen, B., Holzmann, M., Kooistra, W. H., Lara, E., Le Bescot, N., Logares, R., Mahé, F., Massana, R., Montresor, M., Morard, R., Not, F., Pawlowski, J., Probert, I., Sauvadet, A. L., Siano, R., Stoeck, T., Vaulot, D., Zimmermann, P., and Christen, R.: The Protist Ribosomal Reference database (PR2): a catalog of unicellular eukaryote small sub-unit rRNA sequences with curated taxonomy, Nucleic Acid. Res., 41, 597-604, 2013.

Hall, J. A., and Safi, K.: The impact of in situ Fe fertilization on the microbial food web in the Southern Ocean, Deep-Sea Res. II, 48, 2591-2613, 2001.

Hammer, $\varnothing$, Harper, D. A. T., and Ryan, P. D.: PAST: paleontological statistics software package for education and data analysis, Palaeontol. Electr., 4, 1-9, 2001.

Henjes, J., Assmy, P., Klaas, C., and Smetacek, V.: Response of the larger protozooplankton to an iron-induced phytoplankton bloom in the Polar Frontal Zone of the Southern Ocean (EisenEx), Deep Sea Res. I, 54, 774-791, 2007.

Jürgens, K. and Massana, R.: Protistan grazing on marine bacterioplankton, in: Microbial Ecology of the Oceans, edited by: Kirchman, D. L., 2nd Edn., 383-441, 2008.

Ki, J.-S. and Han, M.-S.: Efficient 5' ETS walking from conserved $18 \mathrm{~S}$ rDNA sequences of the dinoflagellates Alexandrium and Akashiwo sanguinea (Dinophyceae), J. Appl. Phycol., 17, 475481, 2005.

Kimura, H. and Naganuma, T.: Thraustochytrids: a neglected agent of the marine microbial food chain, Aquat. Ecosyst. Health Manag., 4, 13-18, 2001.

Kunin, V., Engelbrektson, A., Ochman, H., and Hugenholtz, P.: Wrinkles in the rare biosphere: pyrosequencing errors can lead 
to artificial inflation of diversity estimates, Environ. Microbiol., $12,118-123,2010$.

Landry, M. R., Ondrusek, M. E., Tanner, S. J., Brown, S. L., Costantinou, J., Bidigare, R. R., Coale, K. H., and Fitzwater, S.: Biological response to iron fertilization in the eastern equatorial Pacific (IronEx II). I. Microplankton community abundances and biomass, Mar. Ecol. Prog. Ser., 201, 27-42, 2000.

Lasbleiz, M., Lefèvre, D., Leblanc, K., and Quéguiner, B.: Biological productivity regime and in-situ methods comparison around the Kerguelen Island in the Southern Ocean, this volume.

Lie, A. A. Y., Kim, D. Y., Schnetzer, A., and Caron, D. A.: Smallscale temporal and spatial variations in protistan community composition at the San Pedro Ocean Time-series station off the coast of southern California, Aquat. Microb. Ecol., 2, 93-110, 2013.

López-Garcia, P., Rodriguez-Valera, F., Pedrós-Alió, C., and Moreira, D.: Unexpected diversity of small eukaryotes in deep-sea Antarctic plankton, Nature, 409, 603-607, 2001.

López-Garcia P., Philippe H., Gail F., and Moreira D.: Autochthonous eukaryotic diversity in hydrothermal sediment and experimental microcolonizers at the Mid-Atlantic Ridge, P. Natl. Acad. Sci. USA, 100, 697-702, 2003.

Magurran, A. E.: Measuring biological diversity. Blackwell, Malden, 2004.

Mangot, J. F., Domaizon, I., Taib, N., Marouni, N., Duffaud, E., Bronner, G., and Debroas, D.: Short-term dynamics of diversity patterns: evidence of continual reassembly within lacustrine small eukaryotes, Environ. Microbiol., 15, 1745-1758, 2013.

Martin, J. H. and Fitzwater, S. E.: Iron deficiency limits phytoplankton growth in Antarctic waters, Global Biogeochem. Cy., 4, 5$12,1990$.

Massana, R. and Pedrós-Alió, C.: Unveiling new microbial eukaryotes in the surface ocean, Curr. Opin. Microbiol., 11, 213-218, 2008.

Massana, R., Terrado, R., Forn, I., Lovejoy, C., and Pedrós-Alió, C.: Distribution and abundance of uncultured heterotrophic flagellates in the world oceans, Environ. Microbiol., 8, 1515-1522, 2006.

Medinger, R., Nolte, V., Pandey, R. V., Jost, S., Ottenwalder, B., Schlotterer, C., and Boenigk, J.: Diversity in a hidden world: potential and limitation of next-generation sequencing for surveys of molecular diversity of eukaryotic microorganisms, Mol. Ecol., 19, 32-40, 2010.

Monchy, S., Grattepanche, J. D., Breton, E., Meloni, D., Sanciu, G., Chabe, M., Delhaes, L., Viscogliosi, E., Sime-Ngando, T., and Christaki U.: Microplanktonic community structure in a coastal system relative to a Phaeocystis bloom inferred from morphological and tag pyrosequencing methods, PLoS ONE 7, e39924, doi:10.1371/journal.pone.0039924, 2012.

Myklestad, S. M.: Release of extracellular products by phytoplankton with special emphasis on polysaccharides, Sci. Total Environ., 165, 155-164, 1995.

Nolte, V., Pandey, R.V., Jost, S., Medinger, R., Ottenwälder, B., Boenigk, J., and Schlötterer, C.: Contrasting seasonal niche separation between rare and abundant taxa conceals the extent of protist diversity, Mol. Ecol., 19, 2908-2915, 2010.

Not, F., Valentin, K., Romari, K., Lovejoy, C., Massana, R., Tobe, K., Vaulot, D., and Medlin, L. K.: Picobiliphytes: a marine pi- coplanktonic algal group with unknown affinities to other eukaryotes, Science, 315, 253-255, 2007.

Obernosterer, I., Christaki, U., Lefevre, D., Catala, P., Van Wambeke, F., and LeBaron, P.: Rapid bacterial mineralization of organic carbon produced during a phytoplankton bloom induced by natural iron fertilization in the Southern Ocean, DeepSea Res. II, 55, 777-789, 2008.

Oliver, J. L., Barber, R. T., Smith, W. O., and Ducklow, H. W.: The heterotrophic bacterial response during Southern Ocean Iron Experiment (SOFeX), Limnol. Oceanogr., 49, 2129-2140, 2004.

Park, Y. H., Durand, I., Kestenare, E., Rougier, G., Zhou, M., d'Ovidio, F., Cotté, C., and Lee J. H.: Polar front around the Kerguelen islands: An up-to-date determination and associated circulation of surface/subsurface water. J. Geophys. Res. Oceans, 119, doi10.1002/2014JC010061, 2014.

Pollard, R., Salter, I., Sanders, R., Lucas, M., Moore, C., Mills, R., Statham, P., Allen, J., Baker, A., and Fones, G.: Southern Ocean deep-water carbon export enhanced by natural iron fertilization, Nature, 457, 577-581, 2009.

Potvin, M. and Lovejoy, C.: PCR-based diversity estimates of artificial and environmental 18S rRNA gene libraries, J. Eukaryot. Microbiol., 56, 174-181, 2009.

Poulton, A. J., Moore, M., Seeyave, S., Lucas, M. I., Fielding, S., and Ward, P.: Phytoplankton community composition around the Crozet Plateau, with emphasis on diatoms and Phaeocystis, Deep Sea Res. II, 54, 2085-2105, 2007.

Prokopowich, C. D., Gregory, T. R., and Crease, T. J. : The correlation between rDNA copy number and genome size in eukaryotes, Genome, 46, 48-50, 2003.

Quéguiner, B.: Iron fertilization and the structure of planktonic communities in high nutrient regions of the Southern Ocean, Deep Sea Res. Part II Top. Stud. Oceanogr., 90, 43-54, 2013.

Quéroué, F. and Sarthou, G.: The distribution of dissolved trace elements around the Kerguelen plateau region, unpublished KEOPS2 data.

Quince, C., Lanzen, A., Curtis, T. P., Davenport, R. J., Hall, N., Head, I. M., Read, L. F., and Sloan, W. T.: Accurate determination of microbial diversity from 454 pyrosequencing data, Nat. Methods, 6, 639-641, 2009.

Raghukumar, S.: The role of fungi in marine detrital processes, in: Marine microbiology: facets and opportunities, edited by: Ramaiah, N. E., National Institute of Oceanography, Goa, 91-101, 2004.

Reeder, J. and Knight, R.: The "rare biosphere": a reality check, Nat. Methods, 6, 636-637, 2009.

Saito, H., Suzuki, K., Hinuma, A., Takahashi, O., Fukami, K., Kiyosawa, H., Saino, T., Saino, T., and Tsuda, A.: Responses of microzooplankton to in situ iron fertilization in the Western Subarctic Pacific (SEEDS), Prog. Oceanogr., 64, 223-236, 2005.

Sackett, O., Armand, L., Beardall, J., Hill, R., Doblin, M., Connelly, C., Howes, J., Stuart, B., Ralph, P., and Heraud, P.: Taxon specific responses of Southern Ocean diatoms to Fe enrichment revealed by FTIR microspectroscopy, this volume.

Schloss, P. D., Westcott, S. L., Ryabin, T., Hall, J. R., Hartmann, M., Hollister, E. B., Lesniewski, R. A., Oakley, B. B., Parks, D. H., Robinson, C. J., Sahl, J. W., Stres, B., Thallinger, G. G., Van Horn, D. J., and Weber C. F.: Introducing mothur: open-source, platform-independent, community-supported software for de- 
scribing and comparing microbial communities, Appl. Environ. Microbiol., 75, 7537-7541, 2009.

Schloss, P. D., Gevers, D., and Westcott, S. L.: Reducing the effects of PCR amplification and sequencing artifacts on 16S rRNA-based studies, PLoS ONE, 6, e27310, doi:10.1371/journal.pone.0027310, 2011.

Schnepf, E. and Kühn, S. F.: Food uptake and fine structure of Cryothecomonas longipes sp. nov., a marine nanoflagellate incertae sedis feeding phagotrophically on large diatoms, Helgol. Mar. Res., 54, 18-32, 2000.

Schoemann, V., Becquevort, S., Stefels, J., Rousseau, V., and Lancelot, C.: Phaeocystis blooms in the global ocean and their controlling mechanisms: a review, J. Sea Res., 53, 43-66, 2005.

Skovgaard, A., Massana, R., Balague, V., and Saiz E.: Phylogenetic position of the copepod-infesting parasite Syndinium turbo (Dinoflagellata, Syndinea), Protist, 156, 413-423, 2005.

Smetacek, V., Assmy, P., and Henjes, J.: The role of grazing in structuring Southern Ocean pelagic ecosystems and biogeochemical cycles, Antartct. Sci., 16, 541-558, 2004.
Smetacek, V., Klaas, C., Strass, V.H., Assmy, P., Montresor, M., Cisewski, B., Savoye, N., Webb, A., D' Ovidio, F., Arrieta, J. M., Bathmann, U., Bellerby, R., Berg, G. M., Croot, P., Gonzalez, S., Henjes, J., Herndl, G. J., Hoffmann, L. J., Leach, H., Losch, M., Mills, M. M., Neill, C., Peeken, I., Röttgers, R., Sachs, O., Sauter, E., Schmidt, M. M., Schwarz, J., Terbrüggen, A., and Wolf-Gladrow, D.: Deep carbon export from a Southern Ocean iron-fertilized diatom bloom, Nature, 487, 313-319, 2012.

Tillmann, U., Hesse, K.-J., and Tillmann, A.: Large-scale parasitic infection of diatoms in the North Frisian Wadden Sea, J. Sea Res., 42, 255-261, 1999.

Wolf, C., Frickenhaus, S., Kilias, E. S., Peeken, I., and Metfies, K.: Protist community composition in the Pacific sector of the Southern Ocean during austral summer 2010, Polar Biol., 37, 375-389, 2014.

Zhou, M., Zhu, Y., d'Ovidio, F., Park, Y.H., Durand, I., Kesternare, E., Sanial, E., Van-Beek, P., Quéguiner, B., Carlotti, F., and Blain, F.: Surface currents and upwelling in Kerguelen Plateau regions, 2014.

Zhu, F., Massana, R., Not, F., Marie, D., and Vaulot, D.: Mapping of picoeucaryotes in marine ecosystems with quantitative PCR of the 18S rRNA gene, FEMS Microbiol. Ecol., 52, 79-92, 2005. 